\title{
Digital distance geometry: A survey
}

\author{
P P DAS ${ }^{\dagger}$ and B N CHATTERJI* \\ ${ }^{\dagger}$ Department of Computer Science and Engineering, and \\ * Department of Electronics and Electrical Communication Engineering, \\ Indian Institute of Technology, Kharagpur 721 302, India
}

\begin{abstract}
Digital distance geometry (DDG) is the study of distances in the geometry of digitized spaces. This was introduced approximately 25 years ago, when the study of digital geometry itself began, for providing a theoretical background to digital picture processing algorithms. In this survey we focus our attention on the DDG of arbitrary dimensions and other related issues and compile an up-to-date list of references on the topic.
\end{abstract}

Keywords. Digital space; neighbourhood; path; distance function; metric; digital distance geometry.

\section{Introduction}

Digital geometry (DG) is the study of the geometry of discrete point (or lattice point, grid point) spaces where every point has integral coordinates. In its simplest form we talk about the DG of the two-dimensional (2-D) space which is an infinite array of equispaced points called pixels. Creation of this geometry was initiated in the sixties when it was realized that the digital analysis of visual patterns needed a proper understanding of the discrete space. Since a continuous space cannot be represented in the computer, an approximated digital model found extensive use. However, the digital model failed to capture many properties of Euclidean geometry (EG) and thus the formulation of a new geometrical paradigm was necessary. A similarity can be drawn here between the arithmetic of real numbers and that of integers to highlight the above point. So long we can add, subtract or multiply reals we do not need to devise fresh addition rules or multiplication tables for integers. These are properties which simply extend over a (constrained) digital model. The moment, however, we attempt to divide, a new framework is needed for the integers (say, in the form of well-known floor/ceiling functions). Analogously, some properties of Euclidean geometry extend to the digital case. But the vast majority do not. We tabulate some of them in table 1 .

The study of the above approximate model of EG, is commonly referred to as digital geometry or digital topology. We seek to survey here the notion, definition, characterization and proliferation of distance functions and distance related items in this geometry, under the name of digital distance geometry (DDG). 
Table 1. A few examples of differences between Euclidean and digital geometry.

Euclidean geometry

Properties that

hold

Properties that hold after extension

Properties that do not hold
Euclidean distance is a metric Extendable to higher dimensions

Jordan's theorem in 2-D

Every shortest path which connects two points has a unique mid-point

The shortest path between any pair of points is unique

Only parallel lines do not intersect

Two intersecting lines define an angle between them
Digital geometry

(of the well-known kind)
Euclidean distance is a metric Extendable to higher dimensions

Jordan's theorem in 2-D holds if 4-connectivity is maintained in the object (background) and 8-connectivity in the background (object) $A$ shortest path has a unique midpoint or a mid-point pair

The shortest path between pair of points may not be unique

Lines may not intersect but may not be parallel

Angle is unlikely. Digital trigonometry has been ruled out (Rosenfeld 1983a)

It is true that DG was conceived for two-dimensional image processing and has been serving many of its needs; but it has much potential for application to other fields as well where it can serve in various tasks of data analyses. For example, in applications of $n$-dimensional data processing, attenuation of the huge amount of diverse data in the real space is a practical necessity. A 3-dimensional digital space is used in medical applications like processing of tomographic scan data and also in 3-D object analysis. The study of the 3-D motion along with time requires processing in 4-D. The state-space of the robot arm has 6-dimensions. Higher dimensions result in feature analysis where each property is equivalent to a dimension. Application areas also include computer graphics, robotics, cellular automata theory, interconnection networks and gęometric number theory.

The major theme of the development of DG closely followed the Euclidean geometry with special attention being given to results primarily relevant to image processing and other applications. For example, digital straight lines and curves were characterized and the digital counterpart of Jordan's curve theorem was established. In 3-D, major advances were made in closed surface characterization. In a separate, yet related chain of work, efforts were invested in the study of distance functions relevant in the digital context. Actually it was realized that the Euclidean metric $E_{n}$ was inadequate to deal with the quantized situation. Primarily because $E_{n}$ does not have proper neighbourhood and path definitions in DG and its computation in a digital picture is quite difficult. Thus Cityblock and Chessboard distances in 2-D and grid and lattice distances in 3-D naturally evolved in the initial phase. In spite of these bursts of advancement in DG, there were many deficiencies in various aspects of this geometry some of which have been addressed over the years. For example,

1. Formulation of an axiomatic basis for DG.

2. Definition and study of the discrete geometry of $n-D$ grid-point spaces which was conceived primarily in 2- and 3-D for applications in image analysis. 
3. Execution of a programme of distance geometry (Blùmenthal 1953), which helps to substitute ad hoc notions and definitions by a formal and uniform framework, for the digital space.

In view of the above, the present survey attempts to review the present state-of-theart in DDG. While we elaborate on the major issues of DDG through simple distance forms in $n-D$, special reference is made to $2-\mathrm{D}$ to raise and settle a few fundamental questions of interest to image processing.

\section{Preliminary notions and definitions}

In this section we describe the notions of quantization, and define points, paths, metrics etc. in the context of DG.

\subsection{Tessellations}

An approximate discrete model of the continuous space can be created by partitioning/ tiling the space into well-formed cells/tiles. This partitioning process has been studied for long by mathematicians, under the name of tessellations. By definition, a tessellation is an aggregate of $n$-dimensional cells that cover the $n$-D continuous space without overlapping (Coxeter 1963; Ahuja \& Schachter 1983). Clearly, various arbitrarily shaped cells or tiles can be used for a tessellation. However, compact mathematical treatment needs some uniform and homogeneous properties in a tessellation for it to be practically useful. It has already been proved in Coxeter (1963) that in 2-D there are 11 homogeneous tessellations where the same set of $r$ regular polygons meet at every tessellation vertex (figure 1). These are called the Archimedean tilings of the plane. However only 3 of them are regular in the sense that only one type of regular polygon is used to fill the space. They are the rectangular, hexagonal and triangular tessellations. In contrast to 2-D there is only one regular tessellation - the rectangular one - in 3-D. Surprisingly, 4-D has again three regular tessellations, though all higher dimensions support only the rectangular tessellation as the regular one. Since regularity is a prime necessity for algebraic modelling, the digital geometry of $n$-D space is always restricted to the rectangular partitioning of space. Formally thus, regular rectangular tessellations of the continuous $n$-dimensional space produced by $n$ mutually orthogonal sets of equispaced parallel hyperplanes will be taken as the model here, which can be algebraically represented by $Z^{n}$, where $Z$ is the set of integers. So every cell or digital point $\mathrm{x}=\left(x_{1}, x_{2}, \ldots, x_{n}\right) \in Z^{n}$ is an $n$-tuple of integers. In $2-\mathrm{D}$ we call them pixels, in 3-D voxels, in 4-D rexels and in $n$-D hypervoxels.

As a special case in 2-D, however, digital geometry of triangular and hexagonal grids has also been investigated (e.g. in Bell et al 1989). From the point of view of image analysis, hexagonal digitization in particular has several advantages over the conventional square one. For example, all six neighbours being of the same type, the 4-/8- dichotomy of the square grid is absent here and it also offers a better radial resolution and symmetry. Moreover, it supports simpler parallel architectures and is a better approximation of the human vision grid (Borgefors 1989b, 1992). Yet the hexagonal digitization has not become popular because of the difficulties in hexagonal image acquisition and display and due to the satisfactory performance of the square grid in high resolutions. 
(a)

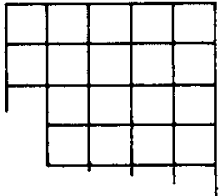

$T_{1}=\left(4^{4}\right)$

rectangular

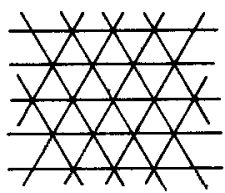

$\mathrm{T}_{2}=\left(3^{6}\right)$

triangular

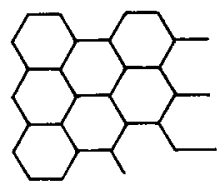

$T_{3}=\left(6^{3}\right)$

hexagonal (b)

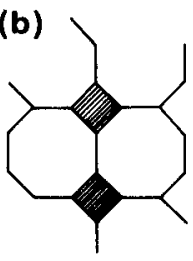

$T_{4}=\left(4 \cdot 8^{2}\right)$

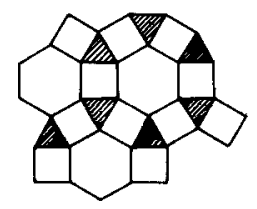

$T_{8}=(3 \cdot 4 \cdot 6 \cdot 4)$

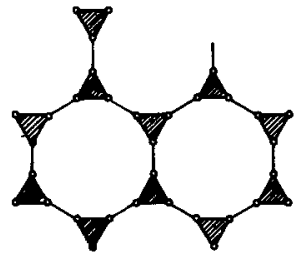

$T_{5}=\left(3 \cdot 12^{2}\right)$

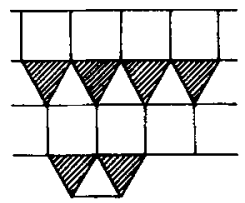

$T_{8}=\left(3^{3} \cdot 4^{2}\right)$

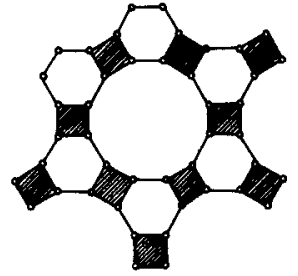

$T_{8}=(4 \cdot 6 \cdot 12)$

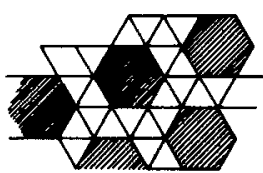

$T_{10}=\left(3^{4} \cdot 6\right)$

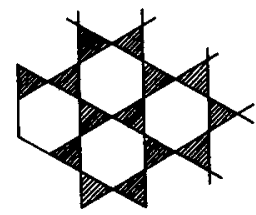

$T_{,}=(3 \cdot 6 \cdot 3 \cdot 6)$

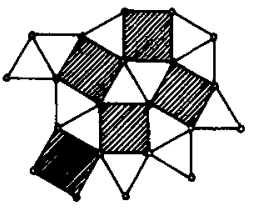

$T_{11}=\left(3^{2} \cdot 4 \cdot 3 \cdot 4\right)$

Figure 1. Archimedean (homogeneous) tilings of the plane. Every tiling is represented by a sequence $T=\left(a_{1}, a_{2}, \ldots, a_{t}\right)$ which specifies the number of sides of the regular polygons that meet at every tessellation vertex. (a) Regular tilings, (b) irregular tilings.

Melter (1986) has developed a set of useful embeddings for all 11 tessellations of the plane in the rectangular grid which can help in constructing special geometries over them. Using Melter's embedding maps any of the homogeneous tessellations can be explored on $Z^{2}$. However no such attempt has yet been reported.

\subsection{Definitions}

In this section we define and explain the most commonly used concepts in DDG.

Integer functions: Two integer functions are often used to express distances in DG. They are the floor and ceiling functions defined below.

$$
\begin{gathered}
\forall x \in R \text {, floor of } x=|\lfloor x\rfloor| \text { and ceiling of } x=|\lceil x\rceil| \text { satisfy } \\
\lfloor x\rfloor,\lceil x\rceil \in Z \text { and } x-1<\lfloor x\rfloor \leqslant x \leqslant\lceil x\rceil<x+1
\end{gathered}
$$

For example, $\lfloor 3 \cdot 2\rfloor=3,\lfloor 4\rfloor=4,\lfloor-5 \cdot 3\rfloor=-6,\lceil 2 \cdot 9\rceil=3,\lceil 7\rceil=7$ and $\lceil-2 \cdot 3\rceil=-2$. Clearly $[-x\rceil=-\lfloor x\rfloor$ and $\lfloor-x\rfloor=-\lceil x\rceil$. We also use round $(x)=\lfloor x+0 \cdot 5\rfloor$ and $x \bmod y=x-y^{*}\lfloor x / y\rfloor$.

Neighbourhood: The neighbourhood $N(\mathbf{x})$ of a point $\mathbf{x}$ is the set of points defined to be neighbours of $\mathbf{x}$. Typically a digital neighbourhood is characterized by a set of 


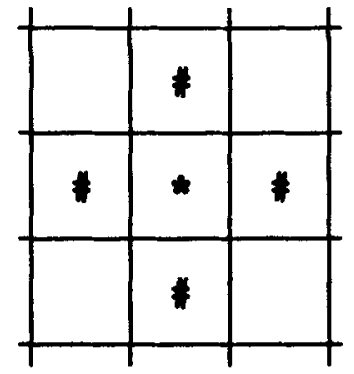

(A)

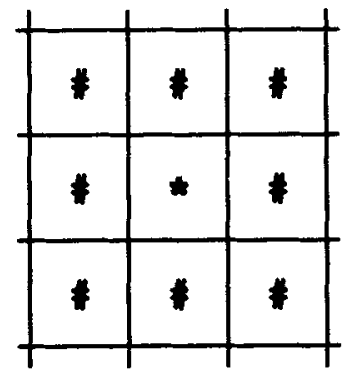

(B)

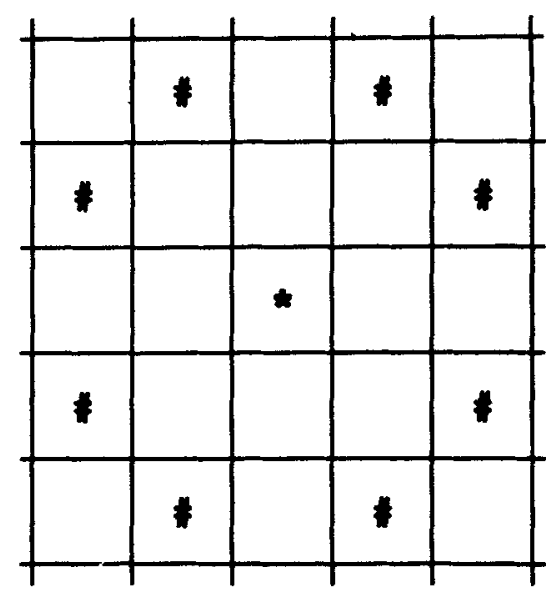

(C)

Figure 2. Few common neighbourhoods in 2-D. Every '\#' denotes a valid neighbour of the central point ' $*$ '. (a) 4-neighbourhood, (b) 8-neighbourhood, (c) knight's-neighbourhood.

difference vectors $\left\{\mathbf{e}^{1}, \mathrm{e}^{2}, \ldots, \mathrm{e}^{k}\right\} \subset Z^{n}$ such that $\forall \mathbf{x} \in Z^{n}, \mathbf{y} \in Z^{n}$ is a neighbour of $\mathbf{x}(\mathbf{y} \in N(\mathbf{x}))$ iff $(x-y) \in\left\{\mathbf{e}^{1}, \mathbf{e}^{2}, \ldots, \mathrm{e}^{k}\right\}$. With every neighbour $\mathrm{e}^{i}$ we also associate a cost $\delta\left(\mathrm{e}^{i}\right)$. Most often this cost is taken to be unity. For example, Cityblock or 4-neighbours, Chessboard or 8-neighbours, and Knight's neighbours are defined by the set of difference vectors $\{( \pm 1,0),(0, \pm 1)\}, k=4,\{( \pm 1,0),(0, \pm 1),( \pm 1, \pm 1)\}, k=8$ and $\{( \pm 1, \pm 2),( \pm 2, \pm 1)\}, k=8$ respectively (figure 2$)$. Common neighbourhoods in 3-D (that is, 6-, 18- and 26-) are shown in figure 3.

Traditionally neighbourhood has often been characterized in DDG by the following five factors:

(i) Proximity: Any two neighbours are proximal sharing a common hyperplane. That is, $\forall i, 1 \leqslant i \leqslant k, \forall j, 1 \leqslant j \leqslant n,\left|e_{j}^{i}\right| \leqslant 1$.

(ii) Separating dimension: The dimension $d$ of the separating hyperplane is bounded by a constant $r$ such that $0 \leqslant r \leqslant d \leqslant n-1$. For example, 4-neighbours have $r=1$ and consequently only line separation is allowed. 8-neighbours, on the other hand, have $r=0$ and both point- and line-separations are allowed. That is, $\forall i$, $1 \leqslant i \leqslant k, n-d=\Sigma_{1 \leqslant j \leqslant n}\left|e_{j}^{i}\right| \leqslant n-r$.

(iii) Separating cost: The cost between neighbours is unity. That is, $\forall i, 1 \leqslant i \leqslant k$, $\delta\left(\mathbf{e}^{i}\right)=1$.

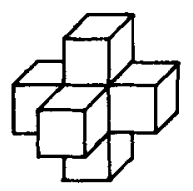

(A)

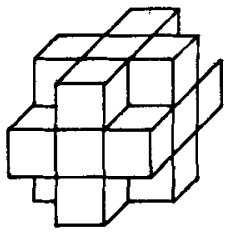

(B)

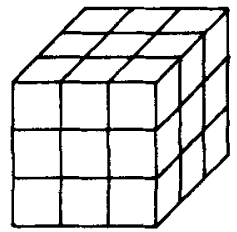

(c)

Figure 3. Few common neighbourhoods in 3-D. (a) 6-neighbourhood, (b) 18neighbourhood, (c) 26-neighbourhood. 
(iv) Isotropy, and symmetry: The neighbourhood is isotropic in all (discrete) directions. That is, $\forall i, 1 \leqslant i \leqslant k, \exists j, 1 \leqslant j \leqslant k$, such that $\mathrm{e}^{j}$ is a permutation and/or reflection of $\mathrm{e}^{i}$.

(v) Uniformity: The neighbourhood relation is identical at all points along a path and at all points of the space. This follows directly from the difference vector definition of neighbourhoods.

Though most distances in DDG follow the above characterization, there are many exceptions where one or more of the above properties are violated.

Path: Given a neighbourhood $N(\cdot)$, a digital path $\pi(u, v)$ connecting two points $u$ and $v$ is defined to be a sequence of points where all pairs of consecutive points are neighbours. That is, $\pi(u, v) \equiv\left\{u=x_{0}, x_{1}, x_{2}, \ldots, x_{M}=v\right\}$ where $x_{i} \in N\left(x_{i+1}\right)$, $0 \leqslant i<M$.

The length of the path $|\pi(u, v)|=\Sigma_{0 \leqslant i<M} \delta\left(x_{i+1}-x_{i}\right)$. For unit cost this is the number of points on the path (excluding either $u$ or $v$ ). Of all paths that connect $u$ to $v$ the one having the smallest length is called the minimal path $\pi^{*}(u, v)$.

Example 1: Let $n=2, N(\cdot)=\{( \pm 1,0),(0, \pm 1),( \pm 1, \pm 1)\}$ - the 8-neighbourhood, $u=(0,0)$ and $v=(9,5)$. So $\pi(u, v)=\{(0,0),(0,1),(1,2),(2,1),(3,1),(4,2),(4,3),(5,3),(6,3)$, $(6,2),(7,1),(8,2),(9,3),(9,4),(9,5)\}$ is a path from u to v. Clearly $|\pi(u, v)|=14$ (figure 4). However this path is not minimal, because a shorter path can be obtained as $\pi_{1}^{*}(u, v)=\{(0,0),(1,1),(2,2),(3,3),(4,4),(5,5),(6,5),(7,5),(8,5),(9,5)\}$ of length $\left|\pi_{1}^{*}(u, v)\right|=9$. Another minimal path (of same length, of course) is $\pi_{2}^{*}(u, v)=\{(0,0)$, $(1,0),(2,0),(3,0),(4,0),(5,1),(6,2),(7,3),(8,4),(9,5)\}$. Also, $\left|\pi_{2}^{*}(u, v)\right|=9$.

Example 2: Let $n=3, N(\cdot)=\{(0,0, \pm 1),(0,+1,0),( \pm 1,0,0),(0, \pm 1, \pm 1),( \pm 1,0, \pm 1)$, $( \pm 1, \pm 1,0)\}-$ the 18-neighbourhood, $u=(2,-7,5)$ and $v=(-8,-4,13)$. Then a

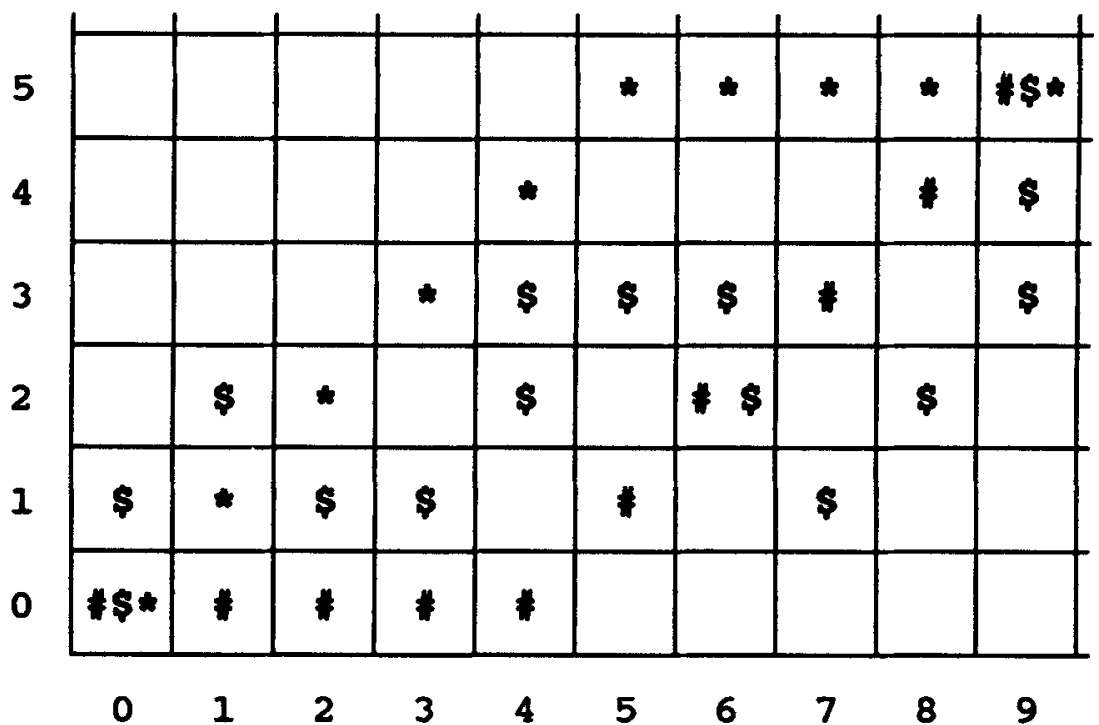

Figure 4. Path and minimal path for 8-neighbourhood in example 1. A nonminimal path $\pi$ is marked with ' $\$$ '. Minimal paths $\pi_{1}$ and $\pi_{2}$ are marked with ' $*$ ' and '\#' respectively. Note that $|\pi|=14$ and $\left|\pi_{1}\right|=\left|\pi_{2}\right|=9$. 


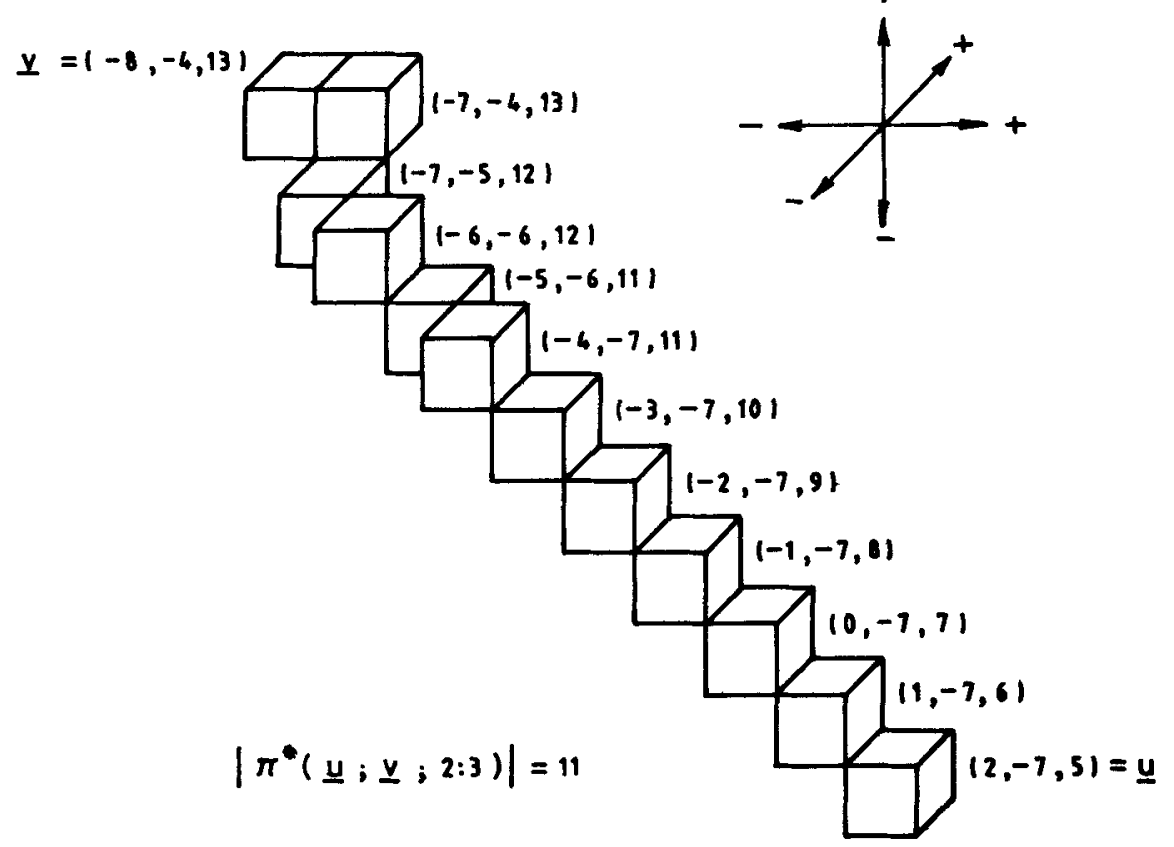

Figure 5. A minimal 18-path in 3-D from $(2,-7,5)$ to $(-8,-4,13)$ in example 2.

minimal path $\pi^{*}(u, v)=\{(2,-7,5),(1,-7,6),(0,-7,7),(-1,-7,8),(-2,-7,9)$, $(-3,-7,10),(-4,-7,11),(-5,-6,11),(-6,-6,12),(-7,-5,12),(-7,-4,13)$, $(-8,-4,13)\}$. The path, as traced above, is shown in figure 5 .

Distance function: The distance $d(u, v)$ between $u, v$ (w.r.t. to a neighbourhood $N(\cdot)$ ) is the length of the shortest path connecting them. That is $d(u, v)=\left|\pi^{*}(u, v)\right|$. It is thus a mapping from $Z^{n} \times Z^{n}$ to $P$, the set of non-negative integers. According to the above neighbourhood definitions we have, $\forall \mathbf{u}, \mathbf{v} \in Z^{n}, d(\mathbf{u}, \mathbf{v})=d(0, \mathbf{u}-\mathbf{v})=d(0,|\mathbf{u}-\mathbf{v}|)$, where 0 is the origin. So we write $d(u, v)=d(\mathbf{x})$, where $\mathbf{x}=|\mathbf{u}-v|$, that is, $\forall i, x_{i}=\left|u_{i}-v_{i}\right|$. Clearly in the 2-D example above, $d(u, v)=9$ and in the $3-D, d(u, v)=11$.

Metric: A distance function $d$ is said to be a metric if it is:

(i) total: $d(u, v)$ is defined and finite,

(note that this is needed because $\forall u, v \in Z^{n}, \pi(u, v)$ may not exist, and hence $d(u, v)$ may not be defined. See, e.g., super-knight's distance in $\$ 6.2 b$ );

(ii) positive: $d(\mathrm{u}, \mathrm{v}) \geqslant 0$;

(iii) definite: $d(\mathbf{u}, \mathbf{v})=0$, iff $\mathbf{u}=\mathbf{v}$;

(iv) symmetric: $d(\mathbf{u}, \mathbf{v})=d(\mathbf{v}, \mathbf{u})$, and;

(v) triangular: $d(\mathbf{u}, \mathbf{v})+d(\mathbf{v}, \mathbf{z}) \geqslant d(\mathbf{u}, \mathbf{z})$; for all $\mathbf{u}, \mathbf{v}, \mathbf{z} \in Z_{n}$.

Euclidean distance: The Euclidean distance $E_{n}$ between $\mathbf{u}$ and $v \in Z^{n}$ is defined as $E_{n}(u, v)=\left(\Sigma_{1 \leqslant i \leqslant n}\left(u_{i}-v_{i}\right)^{2}\right)^{1 / 2}$. We also use $E_{n}(x)=E_{n}(u, v)$ for $x=|u-v|$.

Distance transform (DT): (Borgefors 1992) A distance transformation converts a binary image, consisting of two types of pixels, namely feature (or object - marked 

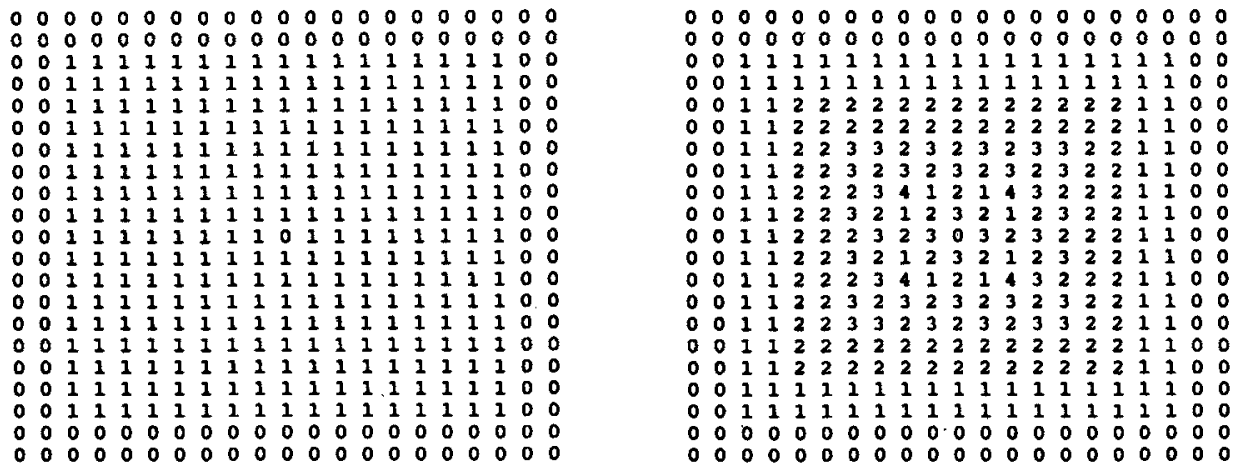

Figure 6. Distance transform of a binary image using knight's distance $(\$ 6.2 \mathrm{~b})$. (a) The binary image with every feature pixel marked as ' 1 ' and every background pixel marked as ' 0 ', (b) knight's DT of the image in (a).

as 1) and non-feature (or background - marked as 0 ), into a distance image where each pixel has a value measuring its distance to the closest non-feature pixels. In figure 6, we show a binary image and its DT. A DT computed with the Euclidean distance is called an Euclidean DT or EDT.

\section{The background}

Since its conception in the later half of the 60's, DDG has gone through three major phases. In the first phase, spanning the period 1966-71, the preliminary concepts of the field were formalized. Subsequently, there was little development in DDG over the next decade (1972-82). Then in the last 10 years many fundamental contributions to DDG have been made. While all readers may not agree with our temporal boundaries there is no denying the fact that there have been three perceptible phases in DDG.

Recent advances in DDG have been surveyed by Kong \& Rosenfeld (1989), by Meiter (1991), and by Borgefors (1992). The first survey (Kong \& Rosenfeld 1989) deals primarily with the topology of the 2-D digital space, and comments on some digital distances in this connection. On the other hand, Melter (1991) considers path-generated-digital-metrics and chamfer distances in two dimensions. He also provides a rich collection of references. Since distance transforms find extensive use in image processing, the algorithms to compute them are of prime importance to DDG. Borgefors (1992) has provided a typically exhaustive review of them without dealing with the mathematical theory of digital distances in depth. Properties of lattice point structures have been discussed in an excellent book by Erdos et al (1989) and several unsolved problems concerning them have earlier been compiled in Hammer (1977).

In contrast to these references, the present one deals primarily with the development of digital distance geometry (DDG) with specific emphasis on the general frameworks for $n$-dimensional DDG and other related issues. In order to restrict the size of the paper, digital distance transforms and a few other topics are only briefly covered here. 


\section{The formative period, (1966-71):}

Formal studies on 2-dimensional digital distance functions, distance computation algorithms and corresponding medial axis transforms were pioneered by Rosenfeld and Pfaltz in the latter half of the 1960's (Rosenfeld \& Pfaltz 1966, 1968; Pfaltz \& Rosenfeld 1967). While Rosenfeld (1970) formalized the notions of 2-D digital connectivity, Rosenfeld \& Pfaltz (1968) introduced the properties of the now well-known Cityblock (Manhattan, Taxicab, Absolute or $d_{4}$ ), Chessboard (King's, Maximum or $d_{8}$ ) and Octagonal $\left(d_{\text {oct }}\right)$ distances and studied their various properties including the closed functional forms given below.

$$
\begin{aligned}
& \forall \mathbf{u}, \mathbf{v} \in Z^{2} \text { and } \mathbf{x}=|\mathbf{u}-\mathbf{v}| \\
& d_{4}(\mathbf{u}, \mathbf{v})=x_{1}+x_{2}, \\
& \left.d_{8}(\mathbf{u}, \mathbf{v})=\max \left(x_{1}, x_{2}\right) \text { (see figure } 4 \text { for an example of } d_{8}\right), \\
& d_{\text {oct }}(\mathbf{u}, \mathbf{v})=\max \left(x_{1}, x_{2},\left\lceil 2\left(x_{1}+x_{2}\right) / 3\right\rceil\right)
\end{aligned}
$$

The Cityblock distance uses 4- (or von-Neumann (Hayes 1984)) neighbours, Chessboard uses 8- (or Moore (Hayes 1984)) neighbours (see figure 2), and the Octagonal distance uses 4- and 8- alternately, along a path starting always with 4-.

A geometric duality between the absolute and the maximum metrics in the real plane was established by Rhodes (1970) as an extension of a self-dual property of the Euclidean metric.

Iterative propagation algorithms were devised in Rosenfeld \& Pfaltz $(1966,1968)$ to efficiently compute digital distances defined above over a subset of digital pictures. The target applications of such distance transforms are the detection of clusters and regularities in a picture and the dissection of a region into "pieces".

In terms of the distance functions, a "skeleton" subset of a digital set is one which minimally describes the digital set. The use of skeletal subsets in computer representation of pictures was studied in Pfaltz \& Rosenfeld (1967). However, the underlying distance being substantially non-Euclidean, the skeletons lacked a number of desirable properties. For instance, the skeletons were not invariant under rotation. To improve the (visual) quality of these skeletons, Montanari (1968) suggested that the problem of obtaining a discrete skeleton be reduced to the determination of optimal paths through a reticular network or graph (and hence, to an optimal policy problem). By increasing the complexity of the network, a "quasi-Euclidean" distance is obtained that approximates the Euclidean distance as closely as desired and thus leads to well-formed skeletons.

The generalization to higher dimensions was first attempted by Mylopoulos \& Pavlidis (1971), where meaningful counterparts of topological notions in quantized spaces were introduced. Specialized Abelian groups were used as a model for such spaces and the notions of dimension, connectivity and order of connectivity were defined formally.

\section{The period of slow progress, (1972-82):}

There was little development in the distance computations on quantized spaces during the 70 's, though the first-ever treatise on digital geometry (Rosenfeld 1979, pp. 7-39; 

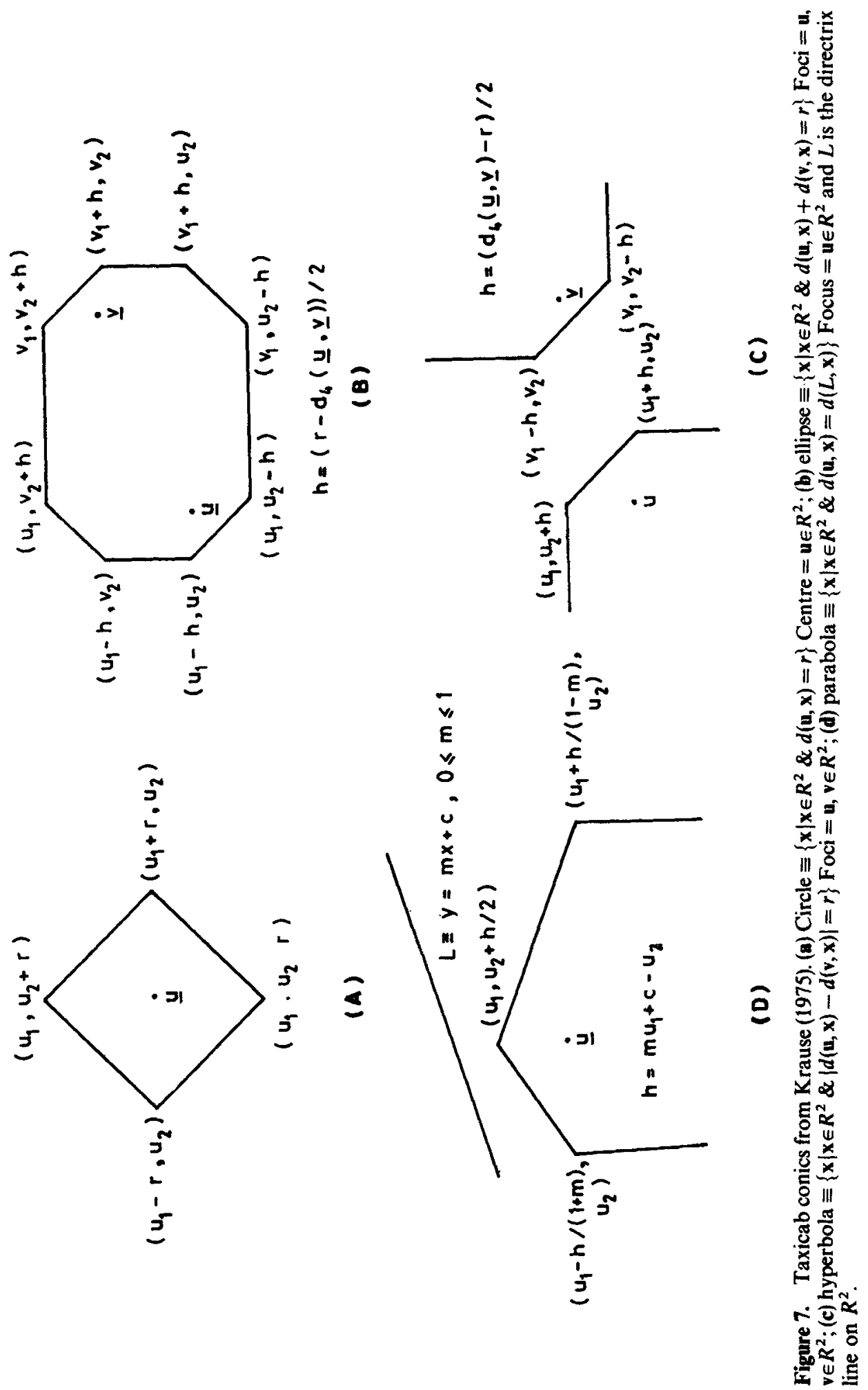
Rosenfeld \& Kak 1982, pp. 209-15) was published by Rosenfeld. The references cited in Rosenfeld (1979) provide a good review of the related topics. Specific issues of geodesics or minimal paths of digital distances were highlighted in Rosenfeld (1978) with notions of path uniqueness.

An interesting study in Taxicab (Cityblock) geometry, which is closely related to the digital geometry, was initiated during the same period (Krause 1975, 1986). The Taxicab geometry, as the name suggests, has wide applications in urban geography for pathplanning in a city. Its Taxicab metric also finds interesting applications in ecology (Schatschneider 1984) for its use in the measurement of "niche overlap" and in the notion of ecological distance between species. Krause $(1975,1986)$ presented a detailed treatment of the Taxicab geometry while elaborating various properties of the equidistant loci of Cityblock and Chessboard distances in depth. For example, see figure 7 for Taxicab circle, ellipse, hyperbola and parabola.

Harary and coworkers (Harary \& Melter 1976; Harary et al 1982) studied the metric dimensions of a graph and analysed their boolean distances from a graphtheoretic point of view. Though not related directly, these papers had substantial influence on further work, particularly in the graph modelling of DDG.

In an attempt to exploit the symmetry of the hexagonal grid, Luczak \& Rosenfeld (1976) looked into the distance properties of such tessellations and formulated the DT algorithms for images represented on them.

In a different direction, Toriwaki and others (Toriwaki et al 1979; Yokoi et al 1981) introduced the distance transformation of a binary line pattern (DTLP) for the extraction of useful features to analyse and recognize given pictorial data. DTLP is a transformation of a binary line pattern by which the value at each feature pixel in an input pattern is changed into the distance measured along a line from that pixel to a suitably chosen edge point. A generalization of DTLP (GDTLP) was also discussed for gray-valued pictures (Toriwaki et al 1982).

It was realized from the very beginning that the computation of the EDT of an image is not an easy task because the Euclidean metric is not amenable to the traditional chamfering used often for distance transforms. Yet the computation of an EDT has been extremely important due to the Euclidean structure of the natural scenes. So, in an attempt to bridge the gap, Danielsson (1980) defined the pseudoEuclidean DT, where most of the pixels in the transformed image get distance values very close to the actual Euclidean distance. The skeletons recovered from such transformations are often close to the natural ones.

\section{The period of major activities, (1983-92):}

A lot of work has been carried out since the early 80's in formalising distances in discrete spaces. Substantial attention has also been focussed on various distance transforms, leading to approximate Euclidean skeletons, and on the algebraic characterization of quantized spaces. A well-organized study has also been carried out in the distance geometry of $n$-dimensional spaces.

\subsection{Graph models in DDG}

Graph-theoretic modelling of digital space has been a popular approach in the study of digital distances because a binary digital image can be conceived as a graph, where 
every pixel represents a node and the adjacency is defined according to the neighbourhood. The cost associated with a neighbour serves as the weight of the corresponding arc. In their work on generalized classes of metrics in $n$-D space, Yamashita \& Ibaraki (1986) have correctly identified this central philosophy of distance computation in the digital domain: "The distance between two points $\mathbf{x}$ and $\mathbf{y}$ is the length of the shortest path connecting $\mathbf{x}$ to $\mathbf{y}$ in an appropriate graph".

In Hübler et al (1982), a 2-dimensional binary image has been viewed as a graph of in-degree and out-degree less or equal to 4 and linear worst case time solutions for several path problems (existence, construction, minimal paths etc.) for such graphs (and hence for binary images) have been discussed. Similar approaches are found in Lipsky (1984) where an $O(n \log n)$ algorithm is devised to decide upon the occurrence of a Manhattan path of alternating horizontal and vertical segments between two given segments. The early works of Melter \& Tomescu (1981a, b) also dealt with similar ideas with special reference to the embeddability of graphs under invariant metric norms. Recently Rosenfeld \& Wu (1989) have reviewed the graph-based notions of digital geometry.

A new class of metrics, called path-generated-digital-metrics (PGDM) has been introduced for the digital plane by Melter \& Tomescu (1983). These metrics actually originate as the minimal path-length between any two nodes of the implicit graph of the plane as defined by a set of elementary unit-length path segments. It has been proved in Melter \& Tomescu (1983) that there are essentially five different metrics in this class, including the classical Cityblock and Chessboard metrics. Subsequently Das (1989b) derived closed form expressions for all five PGDM, explored their disks and deviations from the Euclidean norm and devised an algorithm to trace the minimal PGDM paths. Following the general program of distance geometry (Blumenthal 1953), and an earlier work (Harary \& Melter 1976) on metric dimensions of graphs, Melter and others have also investigated the metric bases relating to the $d_{4}$ and $d_{8}$ distances (Melter \& Tomescu 1984a). They show that unlike the Euclidean distance for which the minimal metric bases for the digital plane are just the set of three non-collinear points, Cityblock and Chessboard distances do not define any finite metric basis for the digital plane. The sizes of minimal metric bases for upright digital rectangles are also derived in Melter \& Tomescu (1984a), and it is shown that there exist rectangles having minimal metric bases of any size $\geqslant 3$. These results, however, are purely theoretical in nature and no possible use for them has been mentioned.

Another important graph-theoretic concept relating to $d_{4}$ and $d_{8}$ distances in 2-D, is about the graphs they induce on the digital plane. Harary et al (1984) characterized the possible metric subgraphs of these Manhattan and King's graphs. A study of the induced graphs of the path-generated metrics particularly with respect to the distancetheoretic properties such as metric bases appeared in Melter \& Tomescu (1984b). Wire-routing in a VLSI layout being isothetic (wires are laid only in horizontal or vertical directions) in nature, characterization of metric subgraphs has applications in circuit extraction and related routing problems.

\subsection{Metrics in 2-D DDG}

Before moving to arbitrary dimensions let us explore a few rich classes of metrics in two dimensions. 
6.2a Simple octagonal distances: The study of distances defined by nonuniform position dependent neighbourhoods was initiated by Rosenfeld \& Pfaltz (1968) when they formulated the Octagonal distance in 2-D. With such a distance, the neighbourhood satisfies all properties except uniformity, and keeps on alternating between 4and 8-types respectively on any path, starting always with a 4-neighbour. So Octagonal distance $d_{\text {oct }}$ can be characterized by a neighbourhood sequence ( $N$-sequence), $B=\{1,2\}$ (where we mark a 4-neighbour as type 1 , and an 8-neighbour as type 2 for uniformity considerations to be explained in $n$-D), which cyclically repeats along the path. Extending the above formulation, Das and others (Das et al 1987b; Das \& Chatterji $1990 \mathrm{~b}$ ) have proposed a generalized (Octagonal) distance function $d(B)$ in $2-\mathrm{D}$, where $B=\{b(1), b(2), \ldots, b(p): b(i) \in\{1,2\}\}$ gives the cyclic sequence of neighbourhood. They derive the following closed functional form for $d(B)$ 's in 2-D.

$$
\begin{aligned}
& \text { For } \mathbf{u}, \mathbf{v} \in Z^{2}, d(\mathbf{u}, \mathbf{v} ; B)=d(\mathbf{x} ; B)=\max \left(d_{1}(\mathbf{x} ; B), d_{2}(\mathbf{x} ; B)\right), \text { where } \mathbf{x}= \\
& |\mathbf{u}-\mathbf{v}|, f(i)=\Sigma_{1 \leqslant j \leqslant i} b(j), g(i)=f(p)-f(i-1)-1, p=|B|, \\
& d_{1}(\mathbf{x} ; B)=\max \left(x_{1}, x_{2}\right), \text { and } \\
& d_{2}(\mathbf{x} ; B)=\Sigma_{1 \leqslant j \leqslant p}\left[\left(\left(x_{1}+x_{2}\right)+g(j)\right) / f(p)\right] .
\end{aligned}
$$

Example 3: $\quad$ Let $B=\{1,2\}$. So, $p=2, f(0)=0, f(1)=1, f(2)=3$ and $g(1)=2, g(2)=1$. Thus $d_{1}(\mathbf{x})=\max \left(x_{1}, x_{2}\right)$ and $d_{2}(\mathbf{x})=\left\lfloor\left(x_{1}+x_{2}+2\right) / 3\right\rfloor+\left\lfloor\left(x_{1}+x_{2}+1\right) / 3\right\rfloor=\left\lceil 2\left(x_{1}+\right.\right.$ $\left.x_{2}\right) / 37$.

Example 4: Let $B=\{1,2\}, \mathbf{u}=(0,0)$ and $\mathbf{v}=(9,5) . \pi(\mathbf{u}, \mathbf{v} ; B) \equiv\{(0,0),(0,1),(1,2),(1,3)$, $(2,4),(3,4),(4,3),(4,2),(5,1),(6,1),(7,1),(7,2),(8,3),(9,3),(9,4),(9,5)\}$ is a path from u to $\mathbf{v}$ determined by $\{1,2\}$. Clearly, $|\pi(\mathbf{u}, \mathbf{v} ; B)|=15$. However, it can be verified from figure 8 that this is not a minimal path, since a minimal path here has length only $=10$. A typical minimal path is $\pi^{*}(\mathbf{u}, \mathbf{v} ; B) \equiv\{(0,0),(1,0),(2,1),(2,2),(3,3),(4,3),(5,4)$, $(6,4),(7,5),(8,5),(9,5)\}$. Note that $d(\mathbf{u}, \mathbf{v})=\left|\pi^{*}(\mathbf{u}, \mathbf{v})\right|=\max (9,5,\lceil 2 \cdot 14 / 3\rceil)=10$.

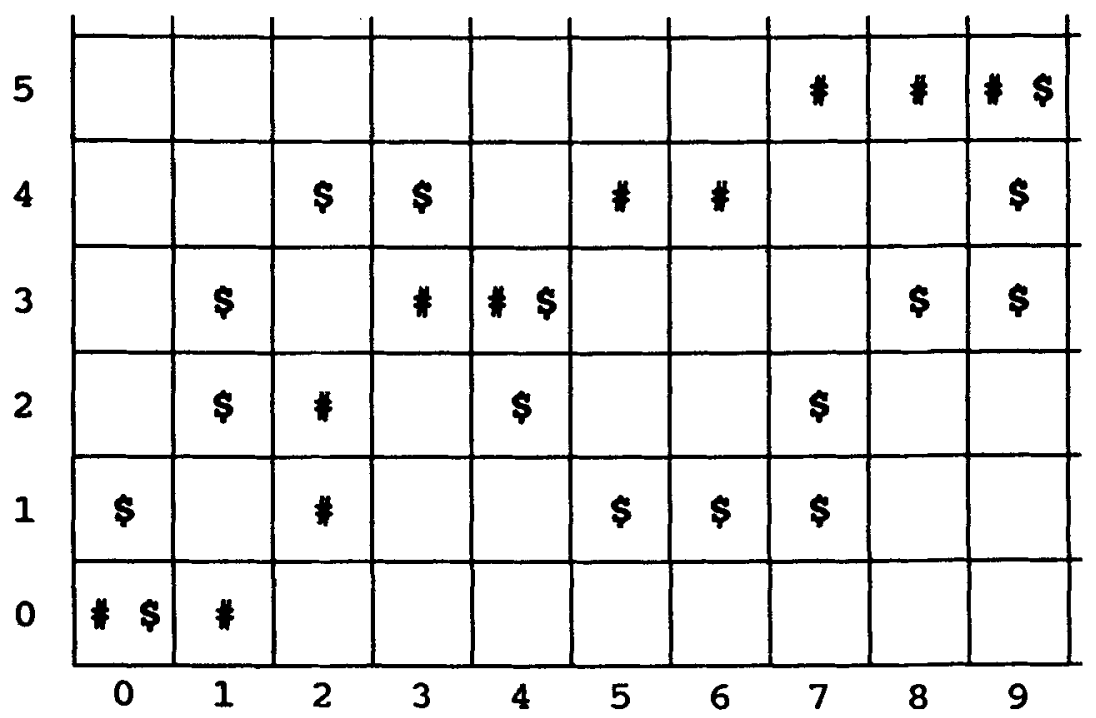

Figure 8. Minimal and non-minimal octagonal paths in example 4. $\pi$ is marked with ' $\$$ ' and $\pi^{*}$ with ' $\#$ '. Note that $|\pi|=15$ and $\left|\pi^{*}\right|=10$. 
It is interesting to note that all $B$ 's do not define $d(B)$ 's that are triangular. For example in $2-D$, if $B=\{2,1\}$, then $(0,0),(1,1)$ and $(2,2)$ would violate the triangularity of $d(B)$ since $d((0,0),(1,1))+d((1,1),(2,2))=1+1<3=d((0,0),(2,2))$. Das and others have proved (Das et al 1987b; Das \& Chatterji 1990b) that

$$
\begin{aligned}
& d(B) \text { is a metric iff B is well-behaved, that is, } \\
& \begin{aligned}
f(i)+f(j) & \leqslant f(i+j), \text { if } i+j \leqslant p ; \\
& \leqslant f(p)+f(i+j-p), \text { if } i+j \geqslant p .
\end{aligned}
\end{aligned}
$$

$H(B ; r)$, the disk of radius $r$ and centre $=0$, is shown to be a digital octagon with corners at $\{( \pm r, \pm c(r)),( \pm c(r), \pm r)\}$ where $c(r)=\lfloor r / p\rfloor(f(p)-p)+f(r \bmod$ $p)-(r \bmod p)$. The asymptotic size of $c(r)$ reflects an important property of the $N$-sequence, which is related to the effective neighbourhood parameter $m_{B}$ defined as $m_{B}=\operatorname{Lim}_{r \rightarrow \infty} c(r) / r+1$. It is shown (Das \& Chatterji 1990b) that $m_{B}=f(p) / p$.

Actually, over a large distance, any $B$ effectively defines a neighbourhood orientation $m_{B}$ which lies between the two extremes of pure horizontal/vertical and pure diagonal moves. The success of the $d(B)$ 's in approximating the Euclidean distance $E_{2}$ is embodied in this effective neighbourhood which can be suitably controlled to derive simple yet powerful metrics.

Out of the class of generalized Octagonal metrics in 2-D which have the same effective neighbourhood value $m_{B}=f(p) / p$ (and hence identical asymptotic behaviour) there exists exactly one metric which has a strikingly simple functional form (involving only one ceiling function). Such distances for which the sum turns out to be a single ceiling function are called Simple Octagonal distances.

It has been proved (Das 1992a) that $d(\mathbf{x} ; B)$ is simple, iff $b(j)=\lfloor j \cdot f(p) / p\rfloor-\lfloor(j-1)$. $f(p) / p\rfloor, 1 \leqslant j \leqslant p$, with $f(p)$ and $p$ relatively prime. A simple $d(B)$ has the functional form $\left.d(\mathbf{x} ; B)=\max \left(\left|x_{1}\right|,\left|x_{2}\right|, \Gamma\left(\left|x_{1}\right|+\left|x_{2}\right|\right) / m\right\rceil\right)$, where $1 \leqslant m \leqslant 2, m=f(p) / p$.

Example 5: Let $p=5$ and $f(p)=7$. So, $b(1)=\lfloor 7 / 5\rfloor-0=1, b(2)=\lfloor 14 / 5\rfloor-\lfloor 7 / 5\rfloor=$ $2-1=1, b(3)=\lfloor 21 / 5\rfloor-\lfloor 14 / 5\rfloor=4-2=2, b(4)=1, b(5)=2$, and $B=\{1,1,2,1,2\}$ is simple with $d(\mathbf{x} ; B)=\max \left(\left|x_{1}\right|,\left|x_{2}\right|,\left\lceil 5\left(\left|x_{1}\right|+\left|x_{2}\right|\right) / 7\right\rceil\right)$.

Using a distance model in the real plane Das (1992a) carries out a detailed analysis to show that both the average relative error and average absolute (normative) error between simple $d(B)$ 's and $E_{2}$ minimizes for $m_{B}=1 \cdot 4=7 / 5$. These findings have also been supported by experiments. Thus no octagonal $d(B)$ in 2-D can perform better than $B=\{1,1,2,1,2\}$ (see the example above). However, $\{1,1,2\},\{1,2\}$ or $\{2\}$ can be used to trade off accuracy for computation.

It is a common notion in 2-D that the Octagonal distance is always greater than the Chessboard distance but smaller than the Cityblock distance. Extending this for further theoretical explorations, Das (1990b) defines a partial ordering between $N$-sequences in 2-D where two $d(B)$ 's (and hence $B$ 's) are ordered, iff $d\left(\mathbf{x} ; B_{1}\right) \geqslant d\left(\mathbf{x} ; B_{2}\right)$, $\forall \mathrm{x} \in Z^{2}$ or $d\left(\mathrm{x} ; B_{1}\right) \leqslant d\left(\mathrm{x} ; B_{2}\right), \forall \mathrm{x} \in Z^{2}$. It has been shown that under this ordering generalized Octagonal distances form a distributive lattice.

6.2b Knight's distance in digital geometry: In the game of chess, the move of a knight has been particularly fascinating. This had earlier led to the well-known problem of knight's tour over a chessboard. Yamashita \& Ibaraki (1986) pointed out that the knight's moves should define a highly interesting and peculiar distance function in two-dimensional digital geometry. The functional form and properties of 
this distance function, called the knight's distance $d_{\mathrm{knight}}$, have been studied by Das $\&$ Chatterji (1988b).

If a knight is placed in the cell $x \in Z^{2}$, then in the next step it can move to any of the eight possible cells $y$ where $(x-y) \in N(2,1)=\{( \pm 1, \pm 2),( \pm 2, \pm 1)\}$ (see figure 2). Thus it is a non-proximal but isotropic and uniform neighbourhood definition involving unity costs. The functional form of the knight's distance $d_{k n i g h t}(u, v)=d_{k n i g h t}(x)$ is given below

$$
\begin{aligned}
\left.d_{\text {knight }}(\mathbf{x})=\max \left(\left\lceil\mid x_{1} / 2\right\rceil, \Gamma\left(x_{1}+x_{2}\right) / 3\right\rceil\right) & +\left(\left(x_{1}+x_{2}\right)\right. \\
& \left.-\max \left(\left\lceil x_{1} / 2\right\rceil,\left\lceil\left(x_{1}+x_{2}\right) / 3\right\rceil\right)\right) \bmod 2,
\end{aligned}
$$$$
\text { if } x \neq(1,0),(2,2) \text {, }
$$$$
=3 \text {, if } x=(1,0) \text {, }
$$$$
=4 \text {, if } \mathbf{x}=(2,2) \text {. }
$$

The authors have also studied the disks of $d_{\text {knight }}$ which are digital octagons with peripheral aberrations (see figure 9 for knight's circle and disk). In addition, they provide minimal path-tracing and DT algorithms for $d_{\text {knight }}$ too.

In an attempt to extend $d_{\text {knieht }}$, Das $\&$ Mukherjee (1990) have defined a super-knight's move via a neighbourhood $N(p, q)=\{( \pm p, \pm q),( \pm q, \pm p)\}, p \geqslant q \geqslant 0$. There are $p, q$ values for which the corresponding $N(p, q)$ distance function is only partial in the sense that there exist $u, v \in Z^{2}$ such that $u$ is not reachable from $v$ using $N(p, q)$ (recall the property (i) that a metric has to be total). For example, $N(1,1)$, a bishop's moves taken in steps of unity, cannot define a metric (a bishop in a black cell can never move to a white cell or vice versa) whereas $N(1,0)$, which is the Cityblock neighbourhood or the rook's moves in unity step, defines a proper metric. Das \& Mukherjee (1990) have characterized a class of neighbourhoods $N(p, q), p \geqslant q \geqslant 0$, to be well-behaved if (1) $p=1, q=0$, or (2) $p+q$ is odd, $q \geqslant 1$ and $p, q$ are mutually prime and it is proved that a super-knight's distance is a metric, iff $N(p, q)$ is well-behaved.

Much remains to be done in such non-isotropic metrics. For example, the formulation
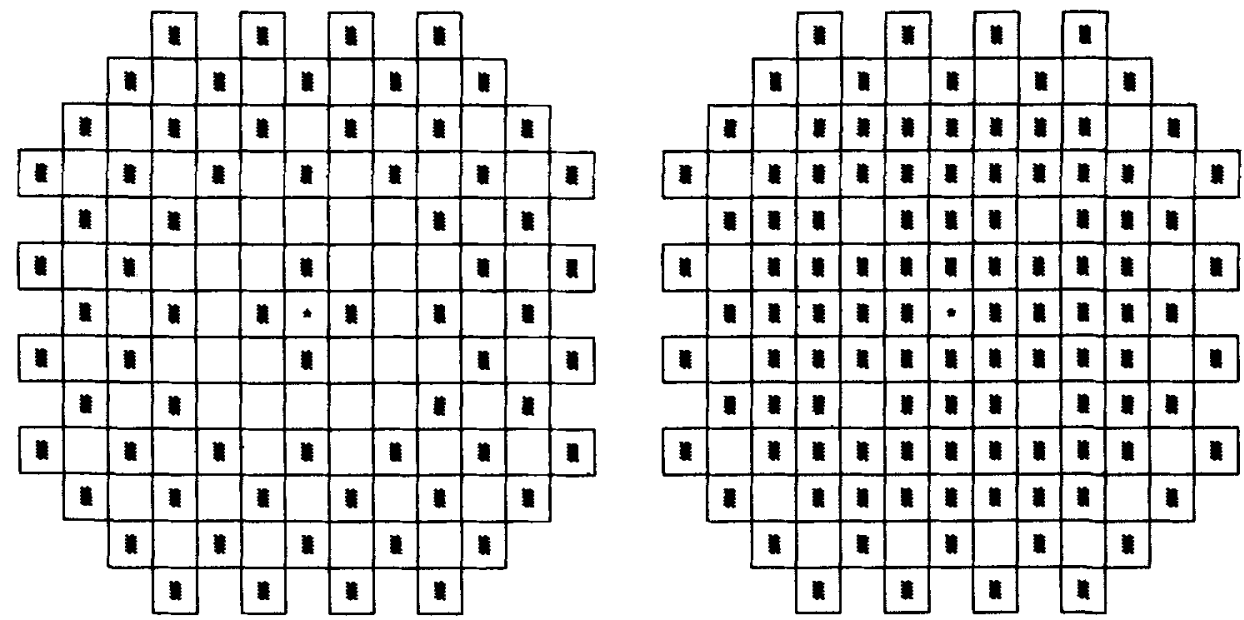

Figure 9. Knight's circle and disk of radius 3 around ' $*$ '. (a) A circle $=\left\{x \mid x \in Z^{2}\right.$ $\& d(*, \mathbf{x})=3\}$, (b) A disk $\equiv\left\{\mathbf{x} \mid \mathbf{x} \in Z^{2} \& d(*, \mathbf{x}) \leqslant 3\right\}$. Every point belonging to the circle/disk is marked as ' 
of a path-tracing algorithm using $N(p, q)$ 's, the study of their disks, and the possible use of these in Euclidean approximation are some of the problems.

\subsection{DDG of arbitrary dimensions}

The study of the distance functions in digitized 3-dimensional space has been pioneered by Okabe et al (1983). Though absolute and maximum metrics in 3-D as grid and lattice distances were introduced by Rosenfeld (1981) earlier, no attempt was made to study the general class of paths, distances and related properties in 3-D. In particular, even the functional form of the $d_{18}$ distance was unknown (Srihari 1981, p. 402) though the fact of its existence prevailed. Since three fundamental types of neighbourhoods are possible in 3-D, namely the 6-, 18- and 26-neighbourhoods (see figure 3 ), the following three metrics arise.

$$
\begin{aligned}
& \forall \mathbf{u}, \mathbf{v} \in Z^{3} \text { and } \mathbf{x}=|\mathbf{u}-\mathbf{v}|, \\
& d_{6}(\mathbf{u}, \mathbf{v})=x_{1}+x_{2}+x_{3},(\text { grid distance) } \\
& d_{18}(\mathbf{u}, \mathbf{v})=\max \left(x_{1}, x_{2}, x_{3},\left\lceil\left(x_{1}+x_{2}+x_{3}\right) / 2\right\rceil\right) \\
& d_{26}(\mathbf{u}, \mathbf{v})=\max \left(x_{1}, x_{2}, x_{3}\right) \text { (lattice distance). }
\end{aligned}
$$

The results of Okabe et al (1983) are actually 3-D generalizations of the 2-D octagonal distance (Rosenfeld \& Pfaltz 1968). Using a shortest path algorithm to trace a minimal path between two voxels, Okabe et al (1983) have derived an explicit function of the coordinates of the terminal voxels which computes the length of the traced minimal path. Different paths were defined by giving different adjacency relations (or neighbourhood sequences as in generalized octagonal distances in 2-D). Seven different distance functions (including the grid, lattice and $d_{18}$ distances) were derived as examples.

Okabe et al (1983) also conjectured an (imperfect) condition on the neighbourhood sequences for the triangularity of such distances which subsequently aroused interest in the study of distances in $n$-dimensions in general. The conjecture was settled in 1984 and a necessary and sufficient condition for the metricity of similarly defined distances in $n$-D was obtained by Yamashita \& Honda (1984). In spite of the fact that the condition presented by them is not computationally attractive - actually a better condition is possible - it provided the necessary impetus to the study of distances in $n$-D digital geometry. As a result, an investigation of the general properties of $n$-D distance functions of digitized spaces has been published (Yamashita \& Ibaraki 1986). These authors assume that a distance between two points is defined as the length of the shortest path connecting them in the underlying graph which is defined by a given cyclic neighbourhood sequence. They compute the distance between any two n-D digital points, given the neighbourhood structure of the path, by solving an equivalent integer programming problem. A necessary and sufficient condition is derived for a neighbourhood sequence to define a distance function satisfying the metric conditions. The important problem of estimating how tight such distances can approximate the Euclidean distance has also been addressed by Yamashita \& Ibaraki (1986) from the point of view of relative and absolute errors and the importance of this work lies in the fact that on the one hand many of the previously known results in lower dimensions can be obtained as special cases, whereas on the other hand it provides a very general formulation for wide classes of distances in $n$-dimensions. 
Moreover this is the first work in DDG which extensively exploits the graph model in an efficient way to derive many important results in DDG. The mathematical rigour of this paper, however, lies beyond the scope of this survey.

In a series of publications following the work of Yamashita and Ibaraki, the present authors studied various digital metrics and their properties in $n-\mathrm{D}$. We summarize this work in the following sections.

6.3a m-Neighbour distance: (Das et al 1987a) The simplest kind of neighbourhood definition in $n$-D satisfies all the desired properties of the neighbourhood relation given in $\$ 2 \cdot 2$. In particular, $\mathbf{u}, \mathbf{v} \in Z^{n}$ are $m$-neighbours, $1 \leqslant m \leqslant n$, iff $0 \leqslant\left|u_{i}-v_{i}\right| \leqslant 1$, $1 \leqslant i \leqslant n$, and $\Sigma_{1 \leqslant i \leqslant n}\left|u_{i}-v_{i}\right| \leqslant m$. Also $\delta(\mathbf{u}-\mathbf{v})=1$. This neighbourhood parameter $m$ relates to the dimensional bound of the separating hyperplane by $m=n-r$. Thus in 2-D, 4- and 8-neighbours have $m=1$ and 2 respectively. In 3-D, 6-, 18- and 26neighbours have $m=1,2$ and 3 respectively. Following the characterization of $m$ neighbours Das et al (1987a) have introduced the $m$-neighbour distance $d_{m}^{n}$ in $n-\mathrm{D}$ as a generalization of 2-D and 3-D metrics, and have proved that

$$
d_{m}^{n}(\mathbf{u}, \mathbf{v})=\left|\pi^{*}(\mathbf{u}, \mathbf{v} ; m: n)\right|=d_{m}^{n}(\mathbf{x})=\max \left(\max _{i} x_{i},\left\lceil\Sigma_{i} x_{i} / m\right\rceil\right)
$$

where $\pi^{*}(u, v ; m: n)$ is a minimal $m$-path corresponding to $d_{m}^{n}$. In traditional notation this means that

$$
d_{1}^{2}=d_{4}, d_{2}^{2}=d_{8}, d_{1}^{3}=d_{6}, d_{2}^{3}=d_{18}, \text { and } d_{3}^{3}=d_{26} .
$$

Also note the similarity between $d_{m}^{2}, m \in\{1,2\}$ and simple octagonal distance with $1 \leqslant m \leqslant 2$.

Example 6: Let $n=4, m=3, \mathrm{u}=(2,1,5,3)$ and $\mathrm{v}=(7,5,9,0)$. Thus a $\pi^{*} \equiv\{(2,1,5,3)$, $(3,2,6,3),(4,3,7,3),(5,3,8,2),(6,4,8,1),(6,5,9,0),(7,5,9,0)\}$ and $\left|\pi^{*}\right|=6$. Also $d_{3}^{4}(u, v)=\max (|2-7|,|1-5|,|5-9|,|3-0|,\lceil(5+4+4+3) / 3\rceil)=\max (5,\lceil 16 / 3\rceil)=6$.

$d_{m}^{n}$ truly generalizes the results of $2-$ and $3-\mathrm{D}$, is a metric in nature and decreases monotonically with increasing $m$. The minimal $m$-paths $\pi^{*}(\mathbf{u}, \mathbf{v} ; m: n)$ corresponding to $d_{m}^{n}$ are non-unique. Hence Das et al (1987a) present an algorithm to trace any one (or all) of them.

During the same time, Klette (1985) also discovered the $m$-neighbour metric while studying the algebraic properties of the $n$-D grid point space. He however did not analyse the properties of this metric in depth.

6.3b t-cost distance: (Das et al 1992) Das and Chatterji have also introduced the cost parameter in $n-\mathrm{D}$ in an uniform way (after the need for doing so was established by Yamashita \& Ibaraki 1986) and defined a new class of metrics $D_{t}^{n}$ called $t$-cost distances (Das et al 1992). For this work they fixed the separating dimensional bound at $r=0$, i.e., all points lying in the unit hypercube around $\mathbf{x}$ are neighbours of $\mathbf{x}$, but changed the unity cost between neighbours. The elementary cost between two neighbours $\mathbf{u}$ and $\mathbf{v}$ is defined in Das et al (1992) as $\delta(\mathbf{u}-\mathbf{v})=\min \left(t, \Sigma_{i}\left|u_{i}-v_{i}\right|\right)$ where $t$ is an integer and $1 \leqslant t \leqslant n$. They prove that $t$-cost distance between $u$ and $v$ is given by

$$
D_{i}^{n}(\mathbf{u}, \mathbf{v})=\Sigma_{1 \leqslant i \leqslant t} f_{i}(\mathbf{u}-\mathbf{v})
$$

where $f_{i}(u)$ is the $i$ th largest component of the vector $|u|$. Interestingly $D_{1}^{n}=d_{n}^{n}$ and 


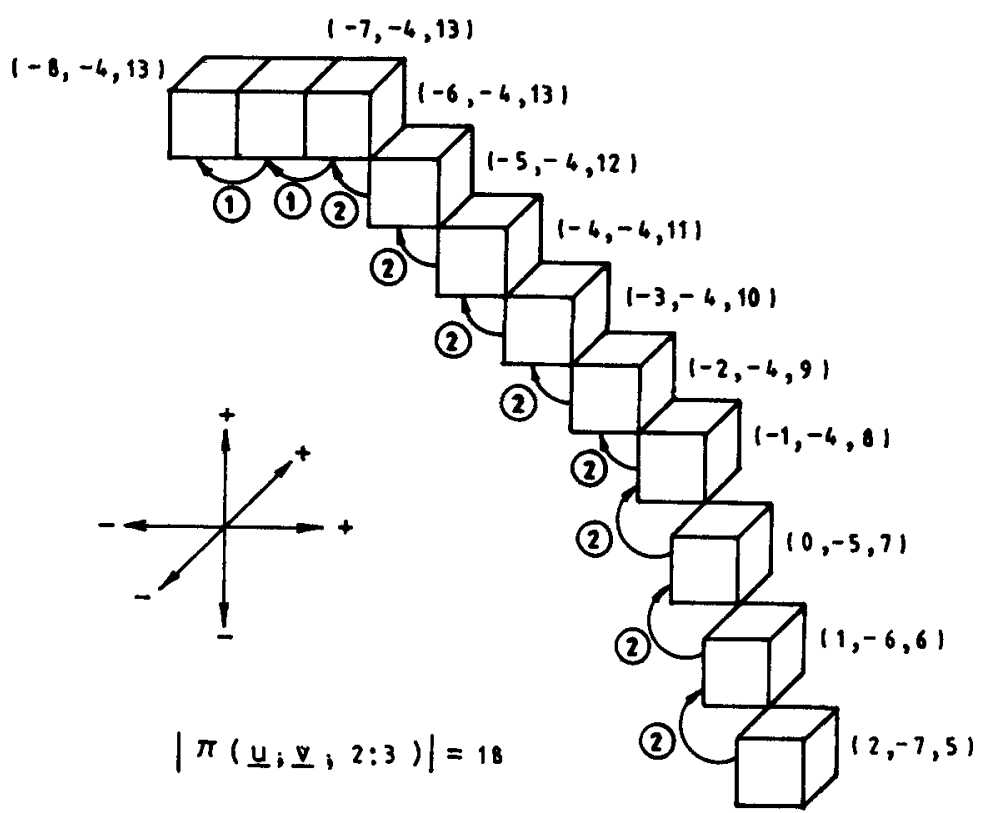

Figure 10. A minimal $t$-cost path in 3-D from $(2,-7,5)$ to $(-8,-4,13)$ with $t=2$ in example 7 . The costs between adjacent points on the path have been encircled in the figure. We have $\left|\pi^{*}\right|=8 \times 2+2 \times 1=18$. Also $D_{2}^{3}((2,-7,5)$, $(-8,-4,13))=D_{2}^{3}((10,3,8))=\max (10,3,8)+\max (\min (10,3), \min (3,8), \min (8,10))$ $=10+8=18$.

$D_{n}^{n}=d_{1}^{n}$. So $D_{2}^{3}$ is a new metric in 3-D given as:

$$
\begin{array}{r}
D_{2}^{3}(\mathbf{u}, \mathbf{v})=D_{2}^{3}(\mathbf{x})=\max \left(x_{1}, x_{2}, x_{3}\right)+\max \left(\min \left(x_{1}, x_{2}\right), \min \left(x_{2}, x_{3}\right),\right. \\
\left.\min \left(x_{3}, x_{1}\right)\right) .
\end{array}
$$

Example 7: Let $n=3, t=2, \mathbf{u}=(2,-7,5)$ and $\mathrm{v}=(-8,-4,13)$. So $\pi^{*}(\mathrm{u}, \mathrm{v})=$ $\{(2,-7,5),(1,-6,6),(0,-5,7),(-1,-4,8),(-2,-4,9),(-3,-4,10),(-4,-4,11)$, $(-5,-4,12),(-6,-4,13),(-7,-4,13),(-8,-4,13)\}$. The path, as traced above, is shown in figure 10 .

$D_{t}^{n}$ thus can be thought of as a generalization in $n$-D from a different perspective. $D_{t}^{n}$ is also a metric and increases with increasing $t$. Minimal $t$-paths, like minimal paths of other digital metrics, are also non-unique and can be computed by an algorithm as in Das et al (1992).

Finally Das and Chatterji (Das et al 1991) combine the $m$-neighbour and $t$-cost definitions to introduce a metric with non-unity cost and $m$-neighbourhood. They call it the $t$-Cost-m-Neighbour $(\mathrm{tCmN})$ distance, $d(t, m: n)$. It generalizes all the properties of $d_{m}^{n}$ and $D_{t}^{n}$ given above, since it satisfies $d(1, m: n) \equiv d_{m}^{n}$ and $d(t, n: n) \equiv D_{t}^{n}$. There are $\left(n^{2}-n+2\right) / 2 \mathrm{tCmN}$ metrics in $n-\mathrm{D}$ and the first unknown one $d(2,3: 4)$ occurs in 4-D.

6.3c Hyperspheres: The introduction of the $m$-neighbour distance has been closely followed by the study of its hyperspheres. Though digital circles and spheres had been analysed earlier in 2- and 3-D, Das \& Chatterji (1990a) made the first attempt in the exploration of hyperspheres in $n-\mathrm{D}$. The hypersphere of $d_{m}^{n}$ of radius $r$ centred at the 
origin is defined as $H(m, n ; r)=\left\{x \mid d_{m}^{n}(\mathbf{x}) \leqslant r\right\}$. Das \& Chatterji (1990a) have shown that $H(m, n ; r)$ is a digital polytope (hyper-cuboctahedron) (Coxeter 1963) with corners at $\phi\left(([, r, \ldots, r, 0,0, \ldots, 0))\right.$ and bounding hyper-surfaces $\left|x_{i}\right|=r$ and $\Sigma_{i}\left|x_{i}\right| / m=r$, where $\phi(\mathbf{x})$ represents the set of all reflections and permutations of $\mathbf{x}$. Using a generating function formulation it has been proved in Das \& Chatterji (1990a) that the volume of $H(m, n ; r)$ (i.e. the no. of digital points in it) is given by an $n$th degree polynomial in $r$ with rational coefficients. For example a Cityblock circle has $|H(1,2 ; r)|=$ $2 r^{2}+2 r+1$ whereas a Chessboard circle has $|H(2,2 ; r)|=4 r^{2}+4 r+1$. An efficient matrix algorithm is also presented to compute these coefficients.

6.3d Errors: The common integer approximations of the Euclidean norm $E_{n}$ either violate triangularity (e.g. $\left\lfloor E_{n}\right\rfloor,\left\lfloor E_{n}+0.5\right\rfloor, E_{n}^{2}$ ) or forbid an easily computable neighbourhood-path definition (e.g. $\left\lceil E_{n}\right\rceil$ ). Hence there have been attempts to use the digital metrics to approximate $E_{n}$. The issue of the approximation by $d_{m}^{n}$ has been studied in depth in Das \& Chatterji (1989). Minimizing the maxima of the relative error between $d_{m}^{n}$ and $E_{n}$, these authors have formulated an algorithm to select the optimal $m=m_{\text {opt }}$ for use in $n$-D. Borgefors (1984) also carried out similar analyses, while minimizing the maxima of the absolute error between $d_{m}^{n}$ and $E_{n}$ normalized w.r.t. the domain (image) size. She conjectured that $\operatorname{Lim}_{n \rightarrow \infty} m_{\mathrm{opt}}=n^{1 / 2}$. This has been confirmed by Das \& Chatterji (1988a). The utility of $D_{t}^{n}$ in approximation is explored in Das et al (1992) to derive a formula for the optimal cost parameter $t_{\text {opt }}$ in $n-D$. Interestingly $t_{\text {opt }} \leqslant 3$ for any $n$. No attempt, however, has so far been made to optimize $m$ and $t$ jointly in $\mathrm{tCmN}$ to get a better approximation of $E_{n}$.

6.3e Distances defined by $N$-sequences: Okabe et al (1983) presented a 3-D extension of the generalized octagonal distances based on neighbourhood sequences (already described in $\$ 6 \cdot 2 \mathrm{a}$ ). (Interestingly the $3-\mathrm{D}$ results were published in 1983 , while the 2-D properties were explored only in 1990). Generalizing Okabe's formulation Das et al (1987b) have proposed a new generalized distance function $d(B)$ in $n$-D, called Hyperoctagonal distance, where $B=\{b(1), b(2), \ldots, b(p): 1 \leqslant b(i) \leqslant n\}$ gives the cyclic sequence of neighbourhood. They derive a closed functional form for $d(B)$ 's in $n$-D. As noted in 2-D, all $N$-sequences do not lead to metric $d(B)$ 's. So, Das et al (1987b) formulated a "well-behaved" condition on a $B$ to test for metricity. This condition is a generalization of the 2-D well-behavedness given earlier and was derived by correcting a conjecture in Okabe et al (1983). They also present an efficient algorithm (Das 1992b) to test the well-behaved property. On specialization following identities, too, were derived.

(i) $d(\{m\})=d_{m}^{n}$ and

(ii) $d(\mathbf{u}, \mathbf{v} ;\{m, m+1\})=d(\mathbf{x})=\max \left(\max _{i} x_{i},\left\lceil 2 \cdot \Sigma_{i} x_{i} /(2 m+1)\right\rceil\right)$ (recollect $d_{\text {oct }}$ where $n=2$ and $m=1)$.

Various $N$-sequence distances satisfying the metric properties, in 2- and 3-D, for sequence length upto 4 and 3 , respectively, have been tabulated in table 2 .

\subsection{Other metrics and related issues}

Besides the metric classes discussed above many other distance functions and distance related geometric properties have been explored. We shall briefly touch upon them in this section. 


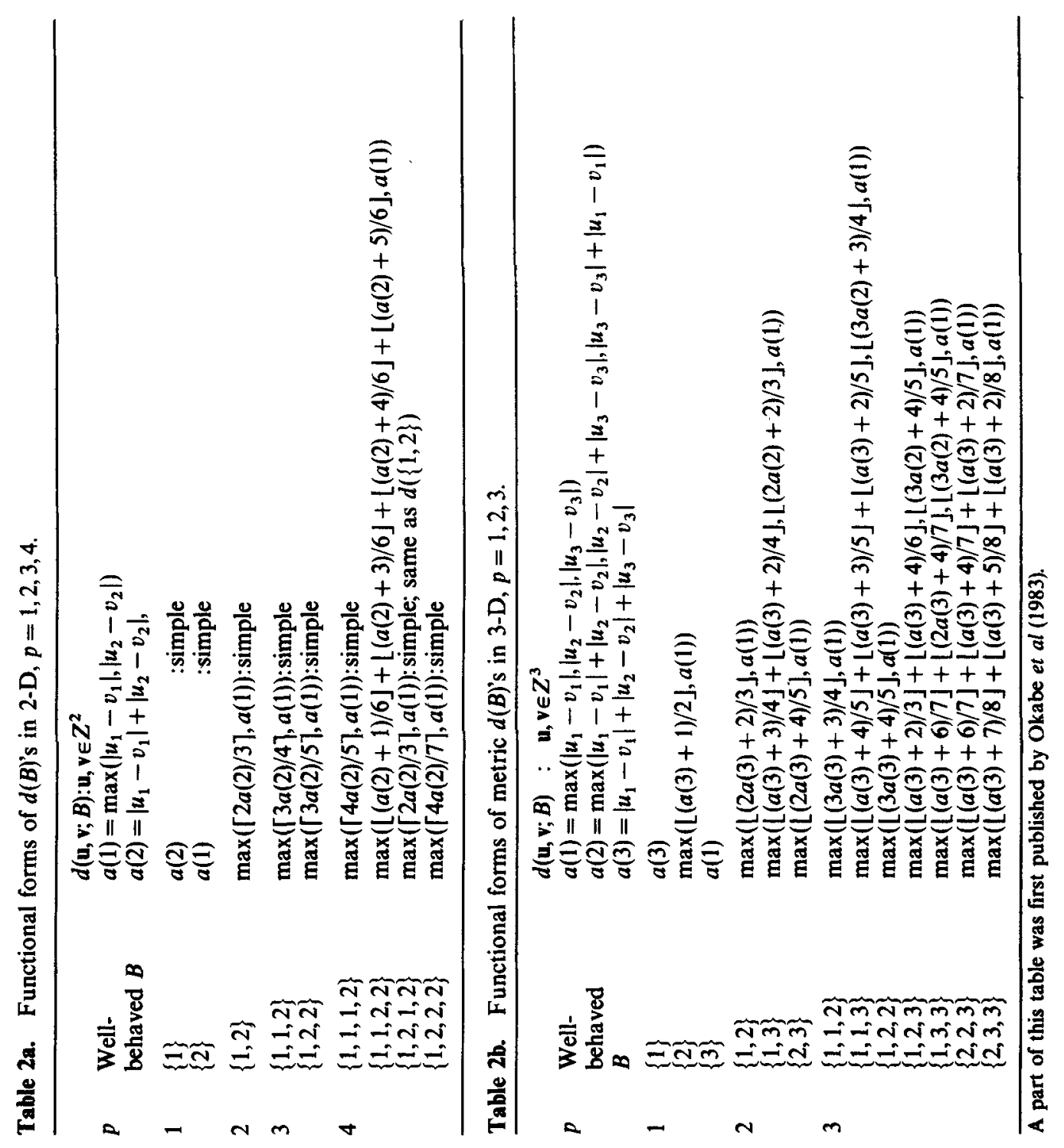


Rosenfeld (1986) defines a function $f$ on digital pictures to be continuous iff $f$ takes neighbours to neighbours, i.e. if $Q$ is a neighbour of $P$ then either $f(Q)=f(P)$ or $f(Q)$ is a neighbour of $f(P)$. He has proved various results for such functions including an almost-fixed-point theorem. Rosenfeld (1983b) has characterized the geometric transforms that have certain simple set theoretic properties. This includes distance and isovist transforms as special cases. Rosenfeld (1987) proves that for any finite connected digital set $S$ and an integer-valued metric on $S$, there exists a non-negative integer $k$ such that, for any $n$ points $p_{1}, p_{2}, \ldots, p_{n}$ of $S$, there exists a point $p$ in $S$ whose average distance from $p_{i}$ 's differs from $k$ by at most $1 / 2$. But unlike the Euclidean case, $k$ is not necessarily unique. He has also defined (Rosenfeld 1985) two distance-derived measures to estimate the sizes of concavities in digital pictures.

A digital triangle has vertices at three lattice points and its sides are the minimal paths between pairs of vertices. Rosenfeld (1983a) shows that many familiar theorems of Euclidean geometry break down for digital triangles.

Melter $(1987,1988)$ also has contributed many important results in 2-D DDG. Using the condition $R$ defined by Rosenfeld (1986) for continuous digital functions, he derives some characterizations for the Cityblock distance in $n$-D. In particular, he presents necessary and sufficient conditions for reflexive and symmetric relations to be associated with the planar Cityblock distance. Further characterizations of both Chessboard and Cityblock metrics have been derived by Rhodes (1990). Using $d_{4}$, Melter (1985) has defined the cover of a digital image as the sum of all distances between pairs of image points. It is shown that certain images can be recognized from their cover values in the presence of additional geometric symmetry conditions. Melter \& Rosenfeld (1989) present a new distance related connectedness which is intermediate between 4 - and 8-connectedness. A set $S$ of lattice points is said to be $(r, s)$-metrically independent, if the congruence of $S$ and $T$ under the $r$-metric implies congruence under the $s$-metric for every digital set $T$. Necessary and sufficient conditions are obtained (Melter \& Wu 1990) for sets to be metrically independent w.r.t. $d_{4}, d_{8}$ and $E_{2}$ metrics.

Due to the multiplicity of minimal paths for digital distances, there have been attempts to count the number of minimal paths between a given pair of points. Path counting has various applications too. For example, it has been used to cluster point sets (Tamura 1982). Rutovitz (1978) had earlier provided two definitions for digital convexity, namely super- and sub-convexity, based on the non-uniqueness of minimal paths. A digital set $S$ is said to be super-(sub-)convex w.r.t. a digital metric $d$ if for all pairs of points $\mathbf{x}, \mathbf{y}$ of $S$, all (at least one) minimal $d$-paths connecting $\mathbf{x}$ to $\mathbf{y}$ lie(s) totally inside $S$. Piper \& Granum (1987) have further elaborated on such notions of convexities while providing an interesting characterization for convex and non-convex domains. Das (1989a), on the other hand, has proposed a matrix algorithm for counting the number of minimal paths constrained by an image subset of the plane. Following the formulations of Rosenfeld \& Pfaltz (1968), Das (1991) has also derived recursive formulae for determining the number of minimal Cityblock, Chessboard or Octagonal paths in the digital plane.

We often need to transform the metric of a metric space into another which satisfies some desirable properties. For example, in the digital space, we want to use an integer equivalent $\left[E_{n}\right\rceil$ of the normal Euclidean distance. We know that while $\left\lceil E_{n}\right\rceil$ preserves the metric properties, $\left\lfloor E_{n}\right\rfloor$ does not. In general a function $f: R^{+} \rightarrow R^{+}$, where $R^{+}$ is the set of non-negative reals, is said to be a Metricity Preserving Transform (MPT) if for any metric space $\langle S, d\rangle, D=f(d)$ is also a metric over $S$. Das (1989c) has 
characterized the metricity preserving transforms deriving the following necessary as well as sufficient condition for metricity preservation.

(i) $f$ is total

(ii) $f(x)=0$, iff $x=0$ and

(iii) $f(x)+f(y) \geqslant \max _{z=|x-y|}^{x+y} f(z) ; \forall x, y \in R^{+}$.

Krause $(1975,1986)$ developed the Taxicab geometry of the real plane where all motions are restricted to horizontal and vertical directions only. Widmayer et al (1987) observed that there are several applications for this geometry to vSLI design (especially in routing). Subsequently the question of diagonal movements was also raised. Widmayer et al (1987) have tackled this by constructing a geometry on a predefined set of fixed orientations. Finally many distance problems have also been solved in this framework. Many existing results then follow as special cases.

Other prominent contributions to DDG during this period include: Shonkwiler's (1989) algorithm for computing the Hausdorff distance in an image in linear time; the extensions of the classical Voronoi diagram for $L_{p}$-metrics in the real plane by Lee (1980) and for digital pictures proposed by Arcelli \& Sanniti di Baja (1986); applications of digital Voronoi diagrams by Toriwaki \& Yokoi (1988, pp. 207-28) to texture analysis; an algorithm by Culberson \& Rudnicki (1989) for constructing trees in optimal time from tree-realizable distance matrices; Das \& Chatterji's (1987) note on the limitations of Chamfer-computability for the Euclidean distance; an efficient algorithm for computing the Manhattan diameter by Du \& Kleitman (1990); a generalized Chamfer algorithm for $m$-neighbour distance transformation (Nag et al 1987); the discrete version (Das 1990a) of Rhodes' (1970) geometric duality theorem; application of DT techniques by Peachey \& Osborne (1990) for quantitative assessment of wall thickness and boundary perimeter in various kinds of wood cells; Rosenfeld and Kong's extensive studies on digital surfaces (Rosenfeld et al 1991) and the design of parallel algorithms by Stojmenovic \& Kim (1987) for solving problems in digital geometry.

Khalimsky (1987) and Kong \& Roscoe (1985) also made significant contributions to digital geometry though they did not use the notion of a metric in their formulation. Bogomolny (1988) has employed various concepts of classical topology to introduce a variant of "digital geometry" which need not be defined on discrete point spaces as in our case.

\subsection{Distance transforms for image processing}

Distance transformations (DT) for images which are subsets of the digital space form a significant part of digital distance geometry. Recently Borgefors (1992) has presented an excellent review of the various DT techniques with a comprehensive and critical comparison of them. We shall merely mention the different algorithms for transformation, and refer the interested reader to Borgefors' article for an informed study.

Most notable amongst the works on DT are the attempts to compute an EDT of a binary picture in 2-D. The first effective sequential propagation algorithm for an Euclidean map was given by Danielsson (1980). Based on a two-component descriptor (a distance label for each point), a map with negligible errors can be produced. 
Yamada (1984) has proposed a parallel algorithm for a "complete" EDT without any error. As a consequence of this "completeness", skeletonization and reverse transformation become simple and systematic. The computational expense of this algorithm is very attractive too, since it takes only the time of an ordinary Chessboard DT to compute the Euclidean DT. Yamada also suggested the use of signed vectors in EDT computation, which has been subsequently adapted by Ye (1988). The advantage of this signed EDT is that each pixel of the transformed image stores a pointer to the feature pixel from which the DT has been computed besides the distance value. This will obviously be helpful in the computation of the Voronoi diagrams and convex hulls, in the detection of dominant points in digital curves etc. Forchhammer (1989) has also computed the exact EDT by first using a weighted integer DT, and then performing a post-processing via table look-up. However his post-correction is possible only for distances upto an upper bound fixed by the mask size and the local distances. Mazille (1989) has outlined a method for getting very good approximations to the EDT by linear filtering of the image. Boult (1990), on the other hand, has elaborated dynamic digital distance maps in two dimensions. Ragnemalm's (1990) thesis also contains a wealth of practical knowledge about the EDT and some of its applications.

Klein (1987) has compared the Euclidean skeletons constructed through an intermediate map of Yamada's (1984) distance with those formed from other methods. He also introduces a more general interpretation of the metric notion that ensures continuity. These Euclidean distance transforms and skeletons have been used by Klein \& Kubler (1987) to give robust characterization of shape and to represent multi-component objects by close approximations to the continuous medial axis. These are also extended for deagglomeration of objects in mutual contact and for the structural interpretation of characteristic patterns in line drawings. Forsgren \& Seideman (1990) have used a medial axis retrieved from discrete distance maps to define a distance measure for estimating similarity of objects. Medial axis from Euclidean distance computation has also been studied by Shih \& Pu (1991).

In an attempt to devise efficient iterative distance transforms for $n$-dimensional digital pictures, Borgefors (1984) has presented an interesting exposition of at least four different families of non-Euclidean distance transforms. These include the $m$-neighbour $\left(d_{m}^{n}\right)$ DT, the hyperoctagonal $(d(B))$ DT and two other chamfer distance transforms. The Euclidean distance is compared with the DT using the upper limit of the difference between the Euclidean and the computed distances. She has also obtained optimal local chamfering distances (steps) to design a suitable approximate Euclidean DT (Borgefors 1983, 1986b). A generalization of Danielsson's (1980) DT in higher dimensions has been carried out under the name of $D$-Euclidean DT. Though the error analyses of this paper are restricted only to four dimensions, it clearly sets a formal framework for the future work in distance transforms in arbitrary dimensions. In a later publication, Borgefors (1986a) developed the theory of optimal chamfer distance transforms in $2-\mathrm{D}$ using local neighbourhood sizes upto $7 \times 7$ pixels. The idea of best integer approximations of optimal real-valued distance transforms has been elaborated. Modifications to these chamfer distance transforms for getting good approximations to the EDT using (almost) integer arithmetic have been suggested by Vossepoel (1988) and Beckers \& Smeulders (1989). Borgefors (1991) further obtained optimum rescaling factors for various integer distance transforms as functions of integer local distances in $3 \times 3$ and $5 \times 5$ neighbourhoods. This generalizes her 
previous solutions (Borgefors 1986a) for "good" distance transforms. Applications of the chamfer DT are also highlighted in Borgefors (1986a). Subsequently these have been used for a fast thinning algorithm to get skeletons very close to the continuous case (Dorst 1986) and also for a pseudo-Euclidean recursive implementation of the Lee algorithm for routing (Dorst \& Verbeek 1986, pp. 917-20). Besides, Borgefors has also treated (Borgefors \& Sanniti di Baja 1988; Borgefors 1989a) distances, distance transforms and skeletons in the hexagonal grid.

Distance transformation of an image being an expensive task, the need for parallel algorithms for computing DT has long been stressed and touched upon in various papers. But probably Borgefors has presented the first effective and efficient algorithm for parallel machines (SIMD and pyramid) (Borgefors 1989c; Borgefors et al 1990). Her algorithm runs in time proportional to the longest side of the image, and uses no arithmetic to compute weighted DT. Ragnemalm (1989) also has implemented a signed EDT algorithm on an SIMD architecture which exploits the parallelism in the neighbourhood operations.

Extending their studies on distance transformation of digitized pictures, Yokoi et al (1981) defined the concept of generalized distance transformation of a binary picture (GDTB) in terms of a sequence of thinning operators with different neighbourhoods. A family of GDTB defined using a sequence of local minimum filtering with the different neighbourhoods was examined in detail. The generalized gray weighted distance transformation (GGWDT) has also been discussed with reference to its inverse transform (IGGWDT). In this connection a new variant, called the max-type DT for binary pictures has been proposed by Suzuki \& Abe (1983). It is a binary equivalent of the DTLP (Toriwaki et al 1979) mentioned in $\$ 5$. This transformation can be compared to the propagation of a wave, which propagates with a constant velocity to the pixels whose connectivity numbers are less than two, and can be applied to extract shape features by measuring the length of the core line such as the major axis of an ellipse. A fast thinning algorithm (Suzuki \& Abe 1986) consisting of the 4-neighbour DT of an input picture, a shrinking operation and post-processing has also been presented by the same authors.

Since the evolution of quadtrees as a compact hierarchical representation for binary images, the distance transforms on recursively decomposed spaces have constituted a salient feature in the study of distance transforms. Such transforms are defined by Samet (1982) using the Chessboard metric. An algorithm is also presented for its computation. Recently, Shaffer \& Stout (1991) have shown that distance transforms can be computed in linear time on images represented by quadtrees. The quadtree medial axis transform (QMAT) has been developed by Samet (1983) and the algorithm for its construction from the quadtree has been designed. Samet (1985) shows that an algorithm for the reconstruction of the quadtree from QMAT can run in an average time proportional to the number of leaf nodes in the quadtree. Another distance measure for a quadtree representation of a binary image was defined by Shneier (1981). This measure depends on the structure of the tree and on the paths through it, rather than on the underlying image represented by it. In some applications such alternative distance measures are preferable to the image-based definitions.

Other major studies in DT include the application of an efficient uniform cost algorithm to DT by Verwer et al (1989) and an algorithm for a generalized distance transformation based on Minkowski operations proposed by Wang \& Bertrand (1988). 


\section{Conclusions}

In this paper we have reviewed the current status in the distance geometry of digital spaces. Though we have compiled most of the literature on DDG till date, our emphasis has been on the studies of paths and distances in $n$-dimensional digital geometry. We have particularly elaborated on a general framework for distance geometry in the digital case. On the one hand, this framework helps to unify various lower dimensional results obtained earlier, and on the other, it generates new interesting classes of metrics in 2-D. Detailed discussion of other important topics like distance transformation in images, digital topology of 2-D space etc., however, could not be taken up here due to the lack of space. The reader may refer to Kong \& Rosenfeld (1989), Melter (1991) and Borgefors (1992) for further in-depth studies.

The authors are extremely grateful to Drs G Borgefors, R A Melter, P P Chakrabarti, N Okabe, E Krause, L Dorst and A Rosenfeld for their continuing support in this work over the past few years. The first author also acknowledges the financial support of the Indian National Science Academy under the grant for Young Scientist Awardees.

\section{References}

Ahuja N, Schachter B J 1983 Tessellations of a plane. In Pattern models (New York: John Wiley \& Sons) chap. 1

Arcelli C, Sanniti di Baja G 1986 Computing Voronoi diagrams in digital pictures. Pattern Recogn. Lett. 4: 383-389

Beckers A L D, Smeulders A W M 1989 A comment on "A note on distance transformations in digital images". Comput. Vision Graphics Image Process. 47: 89-91

Bell S B M, Holroyd F C, Mason D C 1989 A digital geometry for hexagonal pixels. Image Vision Comput. 7: 194-204

Blumenthal L M 1953 Theory and applications of distance geometry (Oxford: Clarendon Press)

Bogomolny A 1988 Digital geometry may not be discrete. Comput. Vision Graphics Image Process. 43: 205-220

Borgefors G 1983 Chamfering: A fast method for obtaining approximations of the Euclidean distancés in $n$ dimensions. Proc. 3rd Scandinavian Conf. Image Analysis Copenhagen, pp. 250-255

Borgefors G 1984 Distance transformations in arbitrary dimensions. Comput. Vision Graphics Image Process. 27: 321-345

Borgefors G 1986a Distance transformations in digital images. Comput. Vision Graphics Image Process. 34: 344-371

Borgefors G 1986b A new distance transformation approximating the Euclidean distance. Proc. 8th Int. Conf. Pattern Recogn. Paris, pp. 336-338

Borgefors G 1989a Distance transformations on hexagonal grid. Pattern Recogn. Lett. 9:97-105

Borgefors G 1989b On using the hexagonal grid, FOA report C 30530-3.4, National Defence Research Institute, Linköping, Sweden, May

Borgefors G 1989c TiME: Time and memory efficient distance transformations for parallel and pyramid machines, FOA report C 30531-3.4, National Defence Research Institute, Linköping, Sweden, May

Borgefors G 1991 Another comment on "A note on distance transformations in digital images". Comput. Vision Graphics Image Process. (Image Understanding) 54: 301-306 
Borgefors G 1992 Distance transforms for digital image processing (under preparation)

Borgefors G, Hartmann T, Tanimoto S L 1990 Parallel distance transformations on pyramid machines: Theory and implementations. Signal Process. 21: 61-68

Borgefors G, Sanniti di Baja G 1988 Skeletonizing the distance transform on the hexagonal grid. Proc. 9th Int. Conf. Pattern Recogn., Rome, pp. 504-507

Boult T E 1990 Dynamic digital distance maps in two dimensions. IEEE Trans. Robotics Autom. 6: 590-597

Culberson J C, Rudnicki P 1989 A fast algorithm for constructing trees from distance matrices. Inf. Process. Lett. 30: 215-220

Coxeter H S M 1963 Regular polytopes (New York: Macmillan)

Danielsson P E 1980 Euclidean distance mapping. Comput. Graphics Image Process. 14: 227-248

Das P P 1989a An algorithm for computing the number of the minimal paths in digital images. Pattern Recogn. Lett. 9: 107-116

Das P P 1989b More on path generated digital metrics. Pattern Recogn. Lett. 10: 25-31

Das P P 1989c Metricity preserving transforms. Pattern Recogn. Lett. 10: 73-76

Das P P 1990a The discrete version of a geometric duality theorem. J. Geometry 38: 23-38

Das P P 1990b Lattices of octagonal distances in digital geometry, Pattern Recogn. Lett. 11: 663-667

Das P P 1991 Counting minimal paths in digital geometry. Pattern Recogn. Lett. 12: 595-603

Das P P 1992a Best simple octagonal distances in digital geometry. J. Approx. Theo. 68: 155-174

Das P P 1992b A note on "Distance functions in digital geometry". Inf. Sci. 64: 181-190

Das P P, Chakrabarti P P, Chatterji B N 1987a Generalized distances in digital geometry. Inf. Sci. 42: 51-67

Das P P, Chakraborti P P, Chatterji B N $1987 \mathrm{~b}$ Distance functions in digital geometry. Inf. Sci. 42: 113-136

Das P P, Chakrabarti P P, Chatterji B N 1991 The t-cost-m-neighbour distance in digital geometry. J. Geometry 42: 42-58

Das P P, Chatterji B N 1987 On chamfer-computability of distances. Proc. Int. Symp. Electronic Devices, Circuits and Systems Kharagpur, pp. 184-186

Das P P, Chatterji B N 1988a A note on "Distance transformations in arbitrary dimensions". Comput. Vision Graphics Image Process. 43: 368-385

Das P P, Chatterji B N 1988b Knight's distance in digital geometry. Pattern Recogn. Lett. 7: 215-226

Das P P, Chatterji B N 1989 Estimation of errors between Euclidean and $m$-neighbour distance. Inf. Sci. 48: 1-26

Das P P, Chatterji B N 1990a Hyperspheres in digital geometry. Inf. Sci. 50: 73-93

Das P P, Chatterji B N 1990b Octagonal distances for digital pictures. Inf. Sci. 50: 123-150

Das P P, Mukherjee J 1990 Metricity of super-knight's distances in digital geometry. Pattern Recogn. Lett. 11: 601-604

Das P P, Mukherjee J, Chatterji B N 1992 The t-cost distance in digital geometry. Inf. Sci. 59: $1-20$

Dorst L 1986 Pseudo-Euclidean skeletons. Proc. 8th Int. Conf. Pattern Recogn., Paris, pp. 286-288

Dorst L, Verbeek P W 1986 The constrained distance transformation: A psuedo-Euclidean, recursive implementation of the Lee algorithm. In Signal Processing III: Theories and applications (eds) I T Young et al (Amsterdam: Elsevier)

Du D Z, Kleitman D J 1990 Diameter and radius in the Manhattan metric. Discrete Comput. Geometry 5: 351-356

Erdös P, Grüber P M, Hammer J 1989 Lattice points (New York: Wiley)

Forchhammer S 1989 Euclidean distances from chamfer distances for limited distances. Proc. 6th Scandinavian Conf. Image Anal. Oulu, Finland, pp. 393-400

Forsgren P O, Seideman P 1990 An interobject distance measure based on medial axis retrieved from discrete distance maps. IEEE Trans. Pattern Anal. Machine Intell. PAMI-12: 390-397

Hammer J 1977 Unsolved problems concerning lattice points (London: Pitman)

Harary F, Melter R A 1976 On the metric dimension of a graph. Ars. Combinatoria 2: 19.1-195

Harary F, Melter R A, Peled U N, Tomescu I 1982 Boolean distance for graphs. Discrete Math. 39: $123-127$

Harary F, Melter R A, Tomescu I 1984 Digital metrics: A graph theoretical approach. Pattern Recogn. Lett. 2: 159-163

Hayes B 1984 Computer recreations. Sci. Am. 250(3): 10-16 
Hübler A, Klette R, Werner G 1982 Shortest path algorithms for graphs of restricted in-degree and out-degree. Elektron. Inf. Kybern. 18: 141-151

Khalimsky E 1987 Topological structures in computer science. J. Appl. Math. Simulation 1: 25-40

Klein F 1987 Euclidean skeletons. Proc. 5th Scandinavian Conf. Image Anal., Stockholm, pp. 443-450

Klein F, Kubler O 1987 Euclidean distance transformation and model guided image interpretation. Pattern Recogn. Lett. 5: 19-29

Klette R 1985 The m-dimensional grid point space. Comput. Vision Graphics Image Process. 30: $1-12$

Kong T Y, Roscoe A W 1985 A theory of binary digital pictures. Comput. Vision Graphics Image Process. 32: 221-243

Kong T Y, Rosenfeld A 1989 Digital topology: Introduction and survey. Comput. Vision Graphics Image Process. 48: 357-393

Krause E F 1975 Taxicab geometry (New York: Dover)

Krause E F 1986 Taxicab geometry, 2nd edn (New York: Dover)

Lee D T 1980 Two-dimensional Voronoi diagrams in the $L_{p}$-metric. J. Assoc. Comput. Mach. 27: 604-618

Lipski W Jr 1984 An $O(n \log n)$ Manhattan path algorithm. Inf. Process. Lett. 19: 99-102

Luczak E, Rosenfeld A 1976 Distance on a hexagonal grid. IEEE Trans. Comput. C-25: 532-533

Mazille J E 1989 Mathematical morphology and convolutions J. Microsc. 156: 3-13

Melter R A 1985 You can (sometimes) tell an image by its cover. Pattern Recogn. Lett. 3: 59-64

Melter R A 1986 Tessellation graph characterization using rosettas. Pattern Recogn. Lett. 4: 79-85

Melter R A 1987 Some characterizations of Cityblock distance. Pattern Recogn. Lett. 6: 235-240

Melter R A 1988 Convexity is necessary - A correction. Pattern Recogn. Lett. 8: 59

Melter R A 1991 A survey of digital metrics. Contemp. Math 119: 95-106

Melter R A, Rosenfeld A 1989 New views of linearity and connectedness in digital geometry. Pattern Recogn. Lett. 10: 9-16

Melter R A, Tomescu 1 1981a Remarks on distances in graphs. ASUI 27: 407-410

Melter R A, Tomescu I 1981 b Isometric embeddability for graphs. Ars Combinatoria 12:111-115

Melter R A, Tomescu I 1983 Path generated digital metrics. Pattern Recogn. Lett. 1:151-154

Melter R A, Tomescu I 1984a Metric bases in digital geometry. Comput. Vision Graphics Image Process. 25: 113-121

Melter R A, Tomescu I 1984b The graphs which correspond to path-generated digital metrics. Proc. Int. Workshop on Graph-theoretic Concepts in Computer Science, Berlin, pp. 278-287

Melter R A, Wu A Y 1990 Metrically independent sets in the digital plane. Inf. Sci. 51:315-329

Montanari U 1968 A method for obtaining skeletons using a quasi-Euclidean distance. J. Assoc. Comput. Mach. 15: 600-624

Mylopoulos J, Pavlidis T 1971 On the topological properties of quantized spaces (I, II). $J$. Assoc. Comput. Mach. 18: 239-254

Nag S, Das P P, Chatterji B N 1987 Generalised chamfer algorithm for $m$-neighbour distance transformation in $n$-D digital pictures. Proc. Int. Symp. Electronic Devices, Circuits and Systems, Kharagpur, pp. 181-183

Okabe N, Toriwaki J, Fukumura T 1983 Paths and distance functions on three dimensional digitized pictures. Pattern Recogn. Lett. 1: 205-212

Peachey T C, Osborne C F 1990 The measurement of wood cell parameters using the distance transform. Wood Fiber Sci. 22: 388-403

Pfaltz J L, Rosenfeld A 1967 Computer representation of planar regions by their skeletons. Commun. ACM 10: 119-125

Piper J, Granum E 1987 Computing distance transformations in convex and non-convex domains. Pattern Recogn. 20: 599-615

Ragnemalm I 1989 The Euclidean distance transform and its implementation on SIMD architectures. Proc. 6th Scandinavian Conf. Image Anal., Oulu, Finland, pp. 379-384

Ragnemalm 11990 Generation of Euclidean distance maps, Linköping Studies in Science and Technology, Thesis No 206, Linköping University, Linköping, Sweden

Rhodes F 1970 A geometric duality for two metrics for the coordinate plane. Math. Gaz. 54: 18-23

Rhodes F 1990 Some characterizations of the Chessboard metric and the Cityblock metric. Pattern Recogn. Lett. 11: 669-675 
Rosenfeld A 1970 Connectivity in digital pictures. J. Assoc. Comput. Mach. 17: 146-160

Rosenfeld A 1978 Geodesics in digital pictures. Inf. Control 36: 74-84

Rosenfeld A 1979 Digital geometry. In Picture languages (New York: Academic Press) chap. 2

Rosenfeld A 1981 Three-dimensional digital topology. Inf. Control 50: 119-127

Rosenfeld A 1983a Some notes on digital triangles. Pattern Recogn. Lett. 1: 147-150

Rosenfeld A 1983b A note on geometric transform of digital sets. Pattern Recogn. Lett. 1: 223-225

Rosenfeld A 1985 Measuring the sizes of concavities. Pattern Recogn. Lett. 3: 71-75

Rosenfeld A 1986 Continuous functions on digital pictures. Pattern Recogn. Lett. 4: 177-184

Rosenfeld A 1987 A note on average distances in digital sets. Pattern Recogn. Lett. 5: 281-283

Rosenfeld A, Kak A C 1982 Digital picture processing 2nd edn (New York: Academic Press) vol. 2

Rosenfeld A, Kong T Y, Wu A Y 1991 Digital surfaces. Comput. Vision Graphics Image Process. (Graphical Models Image Process.) 53: 305-312

Rosenfeld A, Pfaltz J L 1966 Sequential operations in digital picture processing. J. Assoc. Comput. Mach. 13: 471-494

Rosenfeld A, Pfaltz J L 1968 Distance functions on digital pictures. Pattern Recogn. 1: 33-61

Rosenfeld A, Wu A 1989 Digital geometry on graphs. In Computer analysis of images and patterns (eds) K Voss, D Chetverikov, G Sommer (IOS Press)

Rutovitz D 1978 Expanding picture components to natural density boundaries by propagation methods. The notions of fall-set and fall-distance. Proc. 4th Int. Joint Conf. Pattern Recogn., Kyoto, Japan, pp. 657-664

Samet H 1982 Distance transform for images represented by quadtrees. IEEE Trans. Pattern Anal. Mach. Intell. 4: 298-303

Samet H 1983 A quadtree medial axis transform. Commun. ACM 26: 680-693

Samet H 1985 Reconstruction of quadtree from quadtree medial axis transforms. Comput. Vision Graphics Image Process. 29: 311-328

Schatschneider D J 1984 The taxicab group. Am. Math. Mon. 91: 423-428

Shaffer C A, Stout Q F 1991 Linear time distance transform for quadtrees. Comput. Vision Graphics Image Process. (Image Understanding) 54: 215-223

Shih F Y, Pu C C 1991 Medial axis transformation with single-pixel and connectivity preservation using Euclidean distance computation. Proc. 10th Int. Conf. Pattern Recogn. ICPR-B: $723-725$

Shneier M 1981 Path length distances for quadtrees. Inf. Sci. 23: 49-67

Shonkwiler R 1989 An image algorithm for computing the Hausdroff distance efficiently in linear time. Inf. Process. Lett. 30: 87-89

Srihari S N 1981 Representation of three-dimensional digital images. ACM Comput. Surv. 13: $339-424$

Stojmenovic 1, Kim C E 1987 Parallel algorithms for digital geometry, Computer Science Technical Report Cs-87-179, Washington State University

Suzuki S, Abe K 1983 Max type distance transformation for digitised binary pictures and its applications. Trans. IECE Jpn. E-66: 94-101

Suzuki S, Abe K 1986 Sequential thinning of binary pictures using distance transformation. Proc. 8th Int. Conf. Pattern Recogn., Paris, pp. 289-292

Tamura S 1982 Clustering based on multiple paths. Pattern Recogn. 15: 477-483

Toriwaki, Kato N, Fukumura T 1979 Parallel local operations for a new distance transformation of a line pattern and their applications. IEEE Trans. Syst. Man Cybern. SMC-9: 628

Toriwaki J, Tanaka M, Fukumura T 1982 A generalized distance transformation of a line pattern with gray values and its applications. Comput. Vision Graphics Image Process. 20: 319-346

Toriwaki J, Yokoi S 1988 Voronoi and related neighbours on digitised two dimensional space with applications to texture analysis. In Computational morphology (ed.) G Toussaint (Amsterdam: North-Holland)

Verwer B J H, Verbeek P W, Dekker S T 1989 An efficient uniform cost algorithm applied to distance transforms. IEEE Trans. Pattern Anal. Mach. Intell. PAMI-11: 424-429

Vossepoel A M 1988 A note on "Distance transformations in digital images". Comput. Vision Graphics Image Process. 43: 88-97

Wang X, Bertrand G 1988 An algorithm for a generalized distance transformation based on Minkowski operations. Proc. 9th Int. Conf. Pattern Recogn. Rome, pp. 1164-1168 
Widmayer P, Wu Y F, Wang C K 1987 On some distance problems in fixed orientations. SIAM J. Comput. 16: 728-746

Yamada H 1984 Complete Euclidean distance transformation by parallel operation. Proc. 7th Int. Conf. Pattern Recogn. Montreal, pp. 69-71

Yamashita M, Honda N 1984 Distance functions defined by variable neighbourhood sequences. Pattern Recogn. 17: 509-513

Yamashita M, Ibaraki T 1986 Distances defined by neighbourhood sequences. Pattern Recogn. 19: $237-246$

Ye Q -Z 1988 The signed Euclidean distance transform and its applications. Proc. 9th Int. Conf. Pattern Recogn., Rome, pp. 495-499

Yokoi S, Toriwaki J, Fukumura T 1981 On generalized distance transformation of digitized pictures. IEEE Trans. Pattern Anal. Mach. Intell. PAMI-3: 424-443 